\title{
Cutkosky rules and perturbative unitarity in Euclidean nonlocal quantum field theories
}

\author{
Fabio Briscese* \\ SUSTech Academy for Advanced Interdisciplinary Studies, \\ Southern University of Science and Technology, Shenzhen 518055, China and \\ Istituto Nazionale di Alta Matematica Francesco Severi, Gruppo Nazionale di Fisica Matematica, \\ Città Universitaria, P.le A. Moro 5, 00185 Rome, Italy. \\ Leonardo Modestd \\ Department of Physics, Southern University of Science and Technology, Shenzhen 518055, China
}

\begin{abstract}
We prove the unitarity of the Euclidean nonlocal scalar field theory to all perturbative orders in the loop expansion. The amplitudes in the Euclidean space are calculated assuming that all the particles have purely imaginary energies, and afterwards they are analytically continued to real energies. We show that such amplitudes satisfy the Cutkowsky rules and that only the cut diagrams corresponding to normal thresholds contribute to their imaginary part. This implies that the theory is unitary. This analysis is then exported to nonlocal gauge and gravity theories by means of Becchi-Rouet-Stora-Tyutin or diffeomorphism invariance, and Ward identities.
\end{abstract}

\section{INTRODUCTION}

Nonlocal field theories are earning growing interest in the scientific community, since it has been realized that nonlocality might be a key ingredient for the formulation of a quantum renormalizable theory of gravitation. Nonlocal quantum gravity was proposed about 30 years ago by Krasnikov [1] as a superrenormalizable theory for the gravitational interaction, and later extensively studied by Kuz'min (1989) [2] A very general class of local superrenormalizable theories were proposed and extensively studied for the first time in 3. However, only recently have nonlocal $D$-dimensional unitary and superrenormalizable theories been proposed in [4, 5].

Lately, it has been proved that the theory is finite at any perturbative order in the loop expansion [6, 7]. The theory can be formulated in Minkowski or in Euclidean signature, but we cannot pass from one to the other by means of a Wick rotation because of the behavior of the nonlocal form factors at infinity in the complex plane. In fact, the form factors considered here are analytic and do not have poles at finite momenta $k=\left(k^{0}, \vec{k}\right)$, in order to ensure the unitarity of the theory; hence, they must diverge at infinity in some direction on the plane of complex energies $k^{0}$. Therefore, integrals containing such form factors, such as those defining the complex scattering amplitudes, are different when performed along the real or the complex axis, because the integrals on the arcs in the first and fourth quadrant of the $k^{0}$ plane are nonzero. Indeed, even when the same action can be defined both in the Minkowski and Euclidean spaces, the two choices will correspond to two different theories.

In this paper we prove the unitarity of nonlocal theories defined in the Euclidean space. We calculate the complex amplitudes of scattering processes, assuming that all energies $k_{i}^{0}$ and $p_{i}^{0}$ of the loop and external momenta, respectively, are purely imaginary; indeed, all the integrals in the scattering amplitudes are finite, due to the nonlocal form factors. Then, we reconstruct the amplitudes for real values of the external energies by a proper analytic continuation. Finally, we show that the imaginary part of the amplitudes constructed following this procedure is given by Cutkosky rules. Moreover, only cut diagrams corresponding to normal thresholds contribute to such imaginary part, exactly as in the case of local field theories, so that the unitarity of the theory is preserved. In our proof, we do not make use of the reflection positivity in order to avoid any related issues 8 .

We are interested in three main examples of nonlocal field theories: the scalar, gauge, and gravitational nonlocal theories introduced below. However, for the sake of simplicity, we derive Cutkosky rules in the case of a scalar field, and then we discuss how this derivation can be straightforwardly extended to the gauge and gravitational cases.

\footnotetext{
*Electronic address: briscese.phys@gmail.com, briscesef@sustc.edu.com

${ }^{\dagger}$ Electronic address: lmodesto@sustc.edu.com
} 
Nonlocal scalar field theory is described by the following nonlocal Lagrangian:

$$
\mathcal{L}_{\phi}=-\frac{1}{2} \phi e^{H(-\sigma \square)}\left(\square+m^{2}\right) \phi-\lambda \sum_{n=4}^{N} \frac{c_{n}}{n !} \phi^{n},
$$

where $\exp H(-\sigma \square)$ is the nonlocal form factor, $\sigma$ is a parameter with dimensions $[\sigma]=-2$ that fixes the nonlocality scale $\ell_{\Lambda}=\sqrt{\sigma}, N$ is an integer number, and $c_{n}$ are constant parameters. The function $H(z)$ must be entire, i. e. , analytic with no poles at finite $z$, and it is assumed to be a polynomial or an asymptotically logarithmic function in the most interesting cases. The latter class of form factors is need for constructing consistent non-Abelian gauge theories or gravitational theories [see (8) and (9)]. However, in order to prove unitarity, we only need to assume that the form factor does not have zeros and poles at finite values of the loop four-momenta $k$, and that it goes to infinity for $k^{0} \rightarrow \pm i \infty$ and $\vec{k} \rightarrow \pm \infty$, ensuring the finiteness or renormalizability of the scattering amplitudes. Below we study a completely equivalent formulation of the nonlocal scalar field theory, which is obtained from (1) by the following field redefinition:

$$
\phi=e^{-\frac{1}{2} H(-\sigma \square)} \varphi
$$

which recasts (1) in

$$
\mathcal{L}_{\phi}=\frac{1}{2} \partial_{\mu} \varphi \partial^{\mu} \varphi-\frac{1}{2} m^{2} \varphi^{2}-\lambda \sum_{n=4}^{N} \frac{c_{n}}{n !}\left(e^{-\frac{1}{2} H(-\sigma \square)} \varphi\right)^{n} .
$$

The scalar field propagator of (3) is the same as the one of a local two derivative theory, and it satisfies the KallenLechmann representation [9, 10, while nonlocality is restricted to the interaction terms.

Notice that the field redefinition (2) implies a change in the path integral measure. However, such change has no effect on the quantum effective action and on the scattering amplitudes. Indeed, the particular field redefinition (2) changes the integration over the field $\phi$ into an integration over the new field $\varphi$ in the functional integral introducing the Jacobian $\|J\|$ for the transformation

$$
\prod_{x} \frac{\delta \phi(x)}{\delta \varphi(x)}=\prod_{x} \exp -\frac{1}{2} H\left(-\sigma \square_{x}\right)
$$

The transformation (2) is invertible because the form factor $\exp -\frac{1}{2} H(z)$ is never zero for finite values of $z$; hence, one has $\|J\| \neq 0$. Moreover, the Jacobian $\|J\|$ is constant, because it does not depend on the fields; hence, it cancels in the calculation of the normalized generating functional $Z(j) / Z(0)$ (see [2, 11] for more details). In conclusion, the two theories (1) and (3) give the same scattering amplitudes at classical and quantum levels: hence, they are equivalent.

Nonlocal gauge theory was proposed in [2] as a superrenormalizable one, but afterwards a generalization of such theory was shown to be finite in [12, 13]. The action for the finite nonlocal Yang-Mills theory in flat spacetime reads

$$
S_{\mathrm{YM}}=-\frac{1}{2 g_{\mathrm{YM}}^{2}} \int d^{D} x \operatorname{tr}\left[\mathbf{F} e^{H\left(\sigma \mathcal{D}^{2}\right)} \mathbf{F}+s_{g} \sigma^{2} \mathbf{F}^{2}\left(\sigma \mathcal{D}^{2}\right)^{\gamma-2} \mathbf{F}^{2}\right] .
$$

The notation on flat spacetime reads as follows: we use the gauge-covariant box operator defined via $\mathcal{D}_{\Lambda}^{2}=\mathcal{D}_{\mu} \mathcal{D}^{\mu}$, where $\mathcal{D}_{\mu}$ is a gauge-covariant derivative (in the adjoint representation) acting on gauge-covariant field strength $\mathbf{F}_{\rho \sigma}=F_{\rho \sigma}^{a} T^{a}$ of the gauge potential $A_{\mu}$ (where $T^{a}$ are the generators of the gauge group in the adjoint representation.) Moreover, $\sigma=\ell_{\Lambda}^{2}=1 / \Lambda^{2}([\sigma]=2)$, where $\ell_{\Lambda}$ is the nonlocality length scale, $s_{g}$ is a dimensionless parameter, and $\gamma \geqslant 2$ is an integer properly selected in order to have divergences only at one loop. Finally, two special examples of form factors are given in (8) and (9). The same physical scale can be expressed in terms of a length scale $\ell_{\Lambda}$ or an energy scale $\Lambda$.

The action for nonlocal gravity [2, 4, 6] consists in the Einstein-Hilbert term, a nonlocal operator that is quadratic in the Ricci tensor, and a potential at least cubic in the Riemann tensor. The action reads

$$
S_{\mathrm{g}}=-\frac{2}{\kappa_{D}^{2}} \int d^{D} x \sqrt{-g}\left[R+G_{\mu \nu} \gamma(\square) R^{\mu \nu}+V(\mathcal{R})\right] .
$$

where $R, R_{\mu \nu}$, and $G_{\mu \nu}$ are the Ricci scalar, Ricci curvature, and the Einstein tensor, respectively. Moreover, $V(\mathcal{R})$ is a generalized potential and $\mathcal{R}$ stands for scalar, Ricci, or Riemann curvatures, and derivatives thereof. The form factor $\gamma(\square)$ depends on the nonlocality scale $\ell \equiv \sqrt{\sigma}$ and is defined by

$$
\gamma(\square)=\frac{e^{H(\sigma \square)}-1}{\square} .
$$


Two examples of form factors $\exp H(z)$, which are suitable for gravity as well as for gauge theory, are

$$
\begin{aligned}
& H_{\mathrm{K}}(z)=\alpha\left[\log (z)+\Gamma(0, z)+\gamma_{E}\right], \quad \operatorname{Re} z>0, \\
& H_{\mathrm{T}}(p)=\frac{\alpha}{2}\left[\log \left(p^{2}\right)+\Gamma\left(0, p^{2}\right)+\gamma_{E}\right], \quad \operatorname{Re} p^{2}>0,
\end{aligned}
$$

where $\gamma_{E} \approx 0.577216$ is the Euler-Mascheroni constant, $\Gamma(0, x)=\int_{x}^{+\infty} d t e^{-t} / t, p \equiv p(z)$ is a polynomial of $z=-\sigma \square$ of degree $\gamma+1$ with $\gamma>D / 2$, and $\alpha$ is an integer number. Both the entire functions (8) and (9) are asymptotically polynomial in a conical region around the real axis, as required by the locality of the counterterms [6]. Exponential form factors like $\exp \left[(-\square)^{n}\right]$ are suitable for scalar theories, but not for gravity or gauge theories.

The propagators of the gauge and graviton fields are modified by the form factors, yet without introducing any extra poles besides the gauge fields and the graviton. Therefore, in momentum space $\left(k_{\mu}\right)$ the propagators for the theories (5) and (6), respectively, read

$$
\begin{aligned}
& G(k)_{\mu \nu a b}^{\mathrm{YM}}=-i \delta_{a b} \frac{e^{-H\left(\sigma k^{2}\right)}}{k^{2}+i \epsilon}\left(\eta_{\mu \nu}-\frac{k_{\mu} k_{\nu}}{k^{2}}\right)+\left(-\delta_{a b}\right) i \xi_{\mathrm{ym}} \frac{k_{\mu} k_{\nu}}{\omega_{\mathrm{ym}}\left(k^{2}\right)\left(k^{2}+i \epsilon\right)}, \\
& G(k)^{\mathrm{g}}=i \frac{e^{-H\left(\sigma k^{2}\right)}}{k^{2}+i \epsilon}\left(P^{(2)}-\frac{1}{D-2} P^{(0)}\right)+i \frac{\xi_{\mathrm{g}}\left(2 P^{(1)}+\bar{P}^{(0)}\right)}{2\left(k^{2}+i \epsilon\right) \omega_{\mathrm{g}}\left(k^{2} / \Lambda^{2}\right)},
\end{aligned}
$$

where $\xi_{\mathrm{ym}}, \xi_{\mathrm{g}}$ are gauge parameters and $\omega_{\mathrm{ym}}\left(k^{2}\right), \omega_{\mathrm{g}}\left(k^{2}\right)$ are gauge-fixing weighting functions [4]. The tensors $P^{(2)}$, $P^{(0)}, P^{(1)}$, and $\bar{P}^{(0)}$ with four indices (here omitted) are the usual spin-projector operators [4]. The vertices in theories (5) and (6) are very complicated, but not crucial to proving unitarity. For this purpose, it is sufficient to assume that they are analytic functions of the momentum. Moreover, we assume they come from a gauge-invariant or Becchi-Rouet-Stora-Tyutin (BRST)-invariant action. These last two assumptions are surely verified for our theories (5) and (6). Therefore, it is straightforward to derive their unitarity once it is proved for the nonlocal scalar field theory, which does not involve complicated tensorial structure and gauge symmetry.

This paper is organized as follows: in Sec. III we define the complex amplitudes for the Euclidean nonlocal scalar theory (3) and derive the Cutkosky rules. In Sec. III we summarize the relation between unitarity and Cutkosky rules, discussing the issues related to anomalous thresholds. As an application, in Secs IIIA and IIIB we explicitly consider the cases of one- and two-loop diagrams with no anomalous thresholds, proving their unitarity. In Sec. IV we complete the proof of the unitarity of the nonlocal scalar theory, showing that anomalous thresholds do not contribute to the imaginary part of the amplitudes. As an example, in Secs IV A and IV B we prove the unitarity of the triangle and box diagrams, which do have anomalous thresholds. Then, in Sec. V V we discuss how these results are straightforwardly extended to the case of Euclidean nonlocal gauge and gravitational theories, on the basis of BRST invariance and Ward identities. Finally, in Sec. VI we resume these results giving final remarks and conclude.

\section{COMPLEX AMPLITUDES AND CUTKOSKY RULES FOR EUCLIDEAN NONLOCAL SCALAR FIELD THEORY}

In this section, we will define the complex amplitudes for the nonlocal scalar field $(3)$ in the Euclidean space, and we will prove that their imaginary part is given by the Cutkosky rules, which are generalized to the class of theories under consideration.

Since our theory is defined in the Euclidean space, when evaluating the complex amplitudes, the loop integrals are calculated integrating the loop spatial moments in $\mathbb{R}^{3}$ and the loop energies along the imaginary axis $\mathcal{I}$. At the first step, when performing the integrations, also the external energies are taken to be purely imaginary. In this situation, all the poles of the propagators are far from the integration region $\mathcal{I} \times \mathbb{R}^{3}$ in each loop variable, and we can safely integrate, obtaining a finite expression for the amplitudes for purely imaginary energies. Subsequently, these amplitudes are analytically continued to real and positive external energies. Finally, it is proved that the imaginary part of the amplitudes obtained in this way is given by the Cutkosky rules.

For obvious practical limits, the analytic continuation is not obtained from the explicit integrated expressions of the amplitudes. In fact, such explicit expressions would depend on the specific form of the nonlocal vertices. Instead, we continue analytically the generic integral expressions of the amplitudes, considering the limit in which the external energies pass from purely imaginary to real positive values. Indeed, our results will be valid for any nonlocal form factor. However, we must pay special attention in performing such limit, since when we move the external energies from purely imaginary to real positive values, some of the poles of the propagators move through the imaginary axes $\mathcal{I}$. Therefore, the analytic continuation of the amplitudes is obtained deforming $\mathcal{I}$ around these moving poles. This situation is schematically represented in Fig.1. As we will see, for some values of the external energies the new 


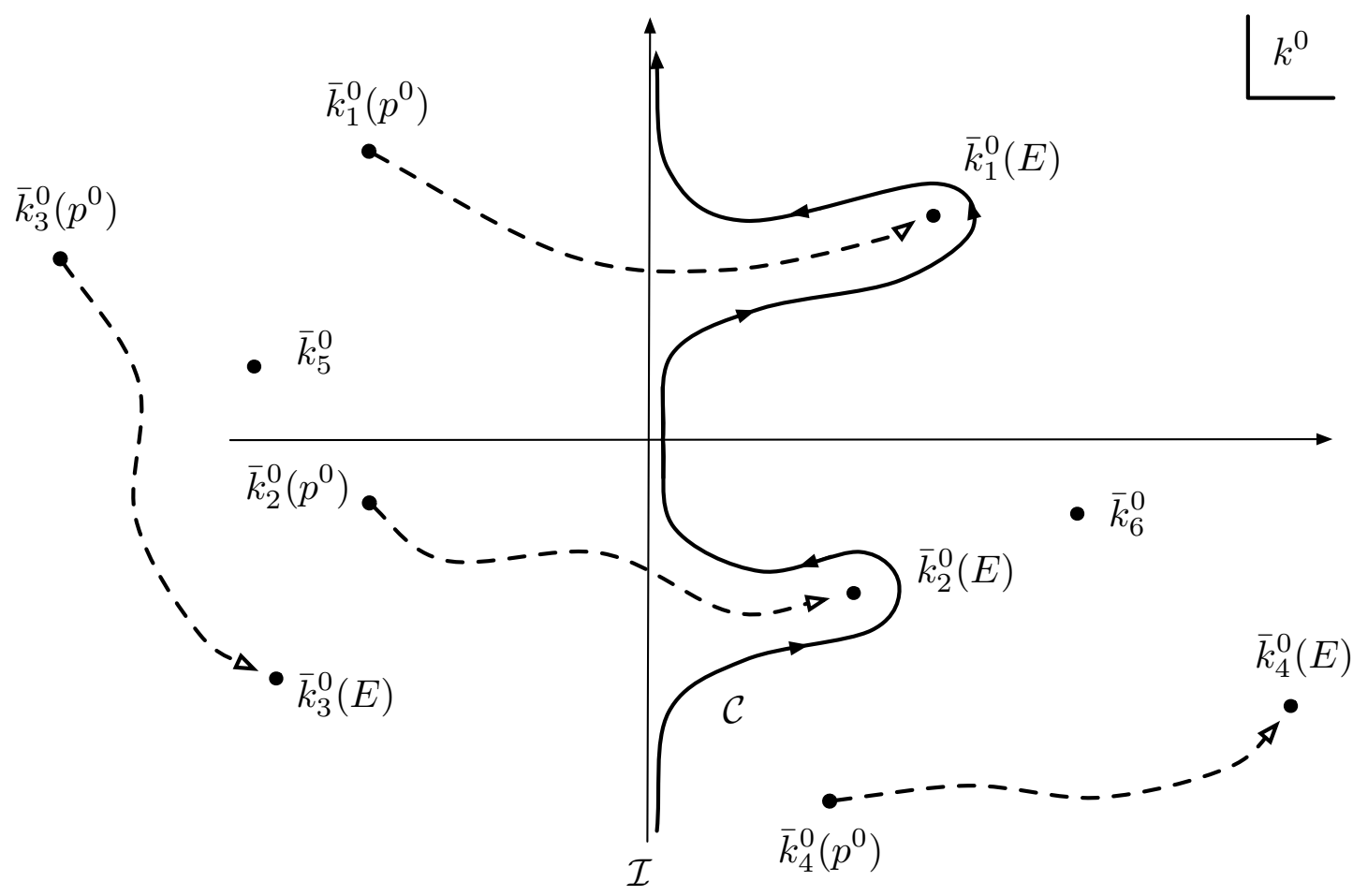

FIG. 1: We schematically plot the poles of the propagators on the $k^{0}$ complex plane, where $k$ is one of the loop momenta. We see that, when the external energy $p^{0}$ is sent to its real and positive value $E$, some pole moves in the $k^{0}$ plane. Some of these moving poles pass through the imaginary axis $\mathcal{I}$, so that the integration contour $\mathcal{C}$ is obtained deforming $\mathcal{I}$ around them.

integration contour $\mathcal{C}$ is pinched by the poles and cannot be deformed further, so that the complex amplitudes become singular.

Let us be more specific, and let us start considering a general scattering amplitude for a process with $L$-loop in the theory (1). For a generic diagram with $I$ internal lines and $V$ vertices, the scattering amplitude will be

$$
i \mathcal{M}=\frac{1}{S_{\#}} \int_{\left(\mathcal{I} \times \mathbb{R}^{3}\right)^{I}} \prod_{i=1}^{I} \frac{d^{4} k_{i}}{(2 \pi)^{4}} \frac{i}{k_{i}^{2}-m^{2}+i \epsilon} \prod_{j=1}^{V}(-i) \lambda \mathcal{V}\left(p^{(j)}\right)(2 \pi)^{4} \delta^{(4)}\left(\sum_{\ell=1}^{N} p_{\ell}^{(j)}\right),
$$

where $\mathcal{I}$ is the imaginary axis, $S_{\#}$ is a combinatoric factor, and the $\delta^{(4)}\left(\sum_{\ell} p_{\ell}^{(j)}\right)$ gives the momentum conservation at the $j$-th vertex. The vertex terms $\mathcal{V}\left(p_{\ell}^{(j)}\right)$ come from the nonlocal interaction at the $j$-th vertex. If we consider a polynomial interaction $\lambda \phi^{N} / N$ ! in (1), corresponding to an interaction term $\lambda(\exp [-H(-\sigma \square) / 2] \varphi)^{N} / N$ ! in (3), the vertex function $\mathcal{V}\left(p_{\ell}^{(j)}\right)$ will be

$$
\mathcal{V}\left(p^{(j)}\right)=\prod_{i=1}^{N} e^{-\frac{H\left(\sigma\left(p_{i}^{(j)}\right)^{2}\right)}{2}},
$$

where $p_{1}^{(j)}, \ldots p_{N}^{(j)}$ are the momenta at the $j$-th vertex. For simplicity, hereafter we will consider vertex functions of the form (13). However, the results presented below are valid for any interaction term of the type given in (1) and (3).

After integration of the delta functions in 12 one has

$$
\mathcal{M}=-\frac{\lambda^{V}}{S_{\#}} \int_{\left(\mathcal{I} \times \mathbb{R}^{3}\right)^{L}} \prod_{i=1}^{L} \frac{i d^{4} k_{i}}{(2 \pi)^{4}} \frac{1}{k_{i}^{2}-m^{2}+i \epsilon} \prod_{j=1}^{I-L} \frac{1}{q_{j}^{2}-m^{2}+i \epsilon} B\left(k_{i}, p_{h}\right),
$$

where $q_{j}$ is a linear combination with coefficients \pm 1 of the loop momenta $k_{i}$ and of the external momenta $p_{h}$. In (14) we have used the topological relation $I-V=L-1$, which implies $i^{I-V}=i^{L-1}$, and we have defined the function 
$B\left(k_{i}, p_{h}\right)$ as

$$
B\left(k_{i}, p_{h}\right) \equiv \prod_{j=1}^{V} \mathcal{V}\left(p_{\ell}^{(j)}\right)
$$

We note that the nonlocality of the theory is encoded only in the term $B\left(k_{i}, p_{h}\right)$, which ensures the ultraviolet convergence of the integrals in the scattering amplitudes. Since by hypothesis $B\left(k_{i}, p_{h}\right)$ has no zeros or poles in the complex hyperplane $\left(\mathbb{C} \times \mathbb{R}^{3}\right)^{L+N}$ for finite momenta $k_{i}$ and $p_{h}$, this function does not introduce other poles in the integrand in (14) than those of the propagators. Indeed, the nonlocality does not change the singularity structure of the scattering amplitude with respect to the case of a local scalar field, and it does not play any role in what we will discuss below.

As explained above, when evaluating (14) we first take purely imaginary values of the external energies $p_{h}^{0}$, so that the poles of the propagators are far from the integration contour. In fact, $k_{i}^{2}-m^{2} \neq 0$ and $q_{j}^{2}-m^{2} \neq 0$ when $k_{i}^{0}$ and $p_{h}^{0}$ are purely imaginary. Afterwards, we consider the analytic continuation of $(14)$, taking the limit in which the external energies go to their physical real values, i.e., $p_{h}^{0} \rightarrow E_{h} \in \mathbb{R}_{0}^{+}$. This is obtained deforming the integration contour $\mathcal{I} \times \mathbb{R}^{3}$ for each loop around the poles that pass through the imaginary axis $\mathcal{I}$ in the limit procedure, but keeping the integration end points to $\pm i \infty$ in order to guarantee the ultraviolet convergence of the loop integrals. ${ }^{1}$

Therefore, the analytic continuation of the amplitude (14) will be given by

$$
\mathcal{M}=-\frac{\lambda^{V}}{S_{\#}} \int_{\left(\mathcal{C}_{L} \times \mathbb{R}^{3}\right)} \int_{\left(\mathcal{C}_{L-1} \times \mathbb{R}^{3}\right)} \ldots \int_{\left(\mathcal{C}_{1} \times \mathbb{R}^{3}\right)} \prod_{i=1}^{L} \frac{i d^{4} k_{i}}{(2 \pi)^{4}} \frac{1}{k_{i}^{2}-m^{2}+i \epsilon} \prod_{j=1}^{I-L} \frac{1}{q_{j}^{2}-m^{2}+i \epsilon} B\left(k_{i}, p_{h}\right),
$$

where $\mathcal{C}_{i} \times \mathbb{R}^{3}$ is the deformed contour for the $i$-th integration variable.

The deformation of the integration contour around the poles piercing the imaginary axis is allowed when such contour is not constrained between separate poles that overlap in the limit procedure in which the external energies go to their real values, and this is always possible whether the Feynman $i \epsilon$ prescription is implemented. On the contrary, when $\epsilon \rightarrow 0$ the integration contour will be constrained between poles that are initially separate but coincide for some real value of the external energies. When this happens, the integration contour cannot be deformed further, and this implies that the amplitude has a singularity at such energies, and develops a branch cut discontinuity. Therefore, the amplitude (16) will have poles and branch cuts at at some values of the external energies in the limit $\epsilon \rightarrow 0$.

In the case of local fields, the singularities of the amplitudes are obtained from the Landau equations [14, which simply express the conditions that two of the propagator poles coincide at some values of the external momenta, so that the integrand in (16) has a double pole; see [10] for a review of the Landau equations. However, since the poles of the local and nonlocal theories are the same, the singularities of the local and nonlocal theories will be the same. Furthermore, the singularities of the amplitudes in nonlocal theories will be determined by the same Landau equations [14 valid for local field theories. Therefore, the nonlocality does not change the positions of the poles of the propagators or the singularity structure of the diagrams.

Having established this fact, we have to show that the imaginary part of the complex amplitudes at the branch cuts corresponding to the Landau poles is given by the Cutkosky rules, as in the local case. Therefore, we have to calculate the limit

$$
\lim _{\epsilon \rightarrow 0}\left\{\mathcal{M}\left(E_{h}, \epsilon\right)-\mathcal{M}^{*}\left(E_{h}, \epsilon\right)\right\}
$$

for the amplitude (16). Since in the original derivation of the Cutkosky rules in [15, Equ. (17) is expressed as the discontinuity of $\mathcal{M}$ at the branch cut, we first prove that, also in the nonlocal case, one has

$$
\lim _{\epsilon \rightarrow 0}\left\{\mathcal{M}\left(E_{h}, \epsilon\right)-\mathcal{M}^{*}\left(E_{h}, \epsilon\right)\right\}=\lim _{\epsilon \rightarrow 0}\left\{\mathcal{M}\left(E_{h}, \epsilon\right)-\mathcal{M}\left(E_{h},-\epsilon\right)\right\} .
$$

Let us consider a specific amplitude $\mathcal{M}_{b a}=\langle b|\mathcal{M}| a\rangle$ obtained by means of the analytic continuation described before as in (14), that is

$$
\mathcal{M}_{b a}\left(p_{h}, \epsilon\right)=-\frac{\lambda^{V}}{S_{\#}} \prod_{i=1}^{L} \int_{-i \infty(\mathcal{C})}^{+i \infty} \int_{\mathbb{R}^{3}} \frac{i d k_{i}^{0} d^{3} k_{i}}{(2 \pi)^{4}} \frac{1}{k_{i}^{2}-m^{2}+i \epsilon} \prod_{j=1}^{I-L} \frac{1}{q_{j}^{2}-m^{2}+i \epsilon} B\left(k_{i}, p_{h}\right),
$$

\footnotetext{
${ }^{1}$ In gravity and gauge theories the convergence is guaranteed by the introduction of other local or nonlocal operators in the action.
} 
where we have explicitly indicated the dependence from the external energies $p_{h}$ and the parameter $\epsilon$. The notation used also indicates that the integration contour $\mathcal{C}$ is oriented from $-i \infty$ to $+i \infty$. The amplitude corresponding to the inverse process $b \rightarrow a$ is given by the same expression as in $\sqrt{19}$, i.e., $\mathcal{M}_{a b}=\mathcal{M}_{b a}$.

The complex conjugate of the amplitude is

$$
\begin{aligned}
\mathcal{M}_{a b}^{*}\left(p_{h}, \epsilon\right) & =-\frac{\lambda^{V}}{S_{\#}}\left[\prod_{i=1}^{L} \int_{-i \infty(\mathcal{C})}^{+i \infty} \int_{\mathbb{R}^{3}} \frac{i d k_{i}^{0} d^{3} k_{i}}{(2 \pi)^{4}} \frac{1}{k_{i}^{2}-m^{2}+i \epsilon} \prod_{j=1}^{I-L} \frac{1}{q_{j}^{2}-m^{2}+i \epsilon} B\left(k_{i}, p_{h}\right)\right]^{*} \\
& =-\frac{\lambda^{V}}{S_{\#}} \prod_{i=1}^{L} \int_{+i \infty\left(\mathcal{C}^{*}\right)}^{-i \infty} \int_{\mathbb{R}^{3}} \frac{-i d k_{i}^{0 *} d^{3} k_{i}}{(2 \pi)^{4}} \frac{1}{\left(k_{i}^{*}\right)^{2}-m^{2}-i \epsilon} \prod_{j=1}^{I-L} \frac{1}{\left(q_{j}^{*}\right)^{2}-m^{2}-i \epsilon} B\left(k_{i}^{*}, p_{h}^{*}\right) .
\end{aligned}
$$

Redefining the name of the integration variable $k_{i}^{*} \rightarrow k_{i}$ one has

$$
\mathcal{M}_{a b}^{*}\left(p_{h}, \epsilon\right)=-\frac{\lambda^{V}}{S_{\#}} \prod_{i=1}^{L} \int_{+i \infty\left(\mathcal{C}^{*}\right)}^{-i \infty} \int_{\mathbb{R}^{3}} \frac{-i d k_{i}^{0} d^{3} k_{i}}{(2 \pi)^{4}} \frac{1}{k_{i}^{2}-m^{2}-i \epsilon} \prod_{j=1}^{I-L} \frac{1}{\tilde{q}_{j}^{2}-m^{2}-i \epsilon} B\left(k_{i}, p_{h}^{*}\right),
$$

where the four vector $\tilde{q}_{i}$ are obtained by the replacement $p^{0} \rightarrow\left(p^{0}\right)^{*}$ in $q_{i}$. The integration contour $\mathcal{C}^{*}$ is obtained from $\mathcal{C}$ by reflection with respect to the real axis, and it is orientated from $+i \infty$ to $-i \infty$. Moreover, the complex conjugation changed the Feynman $i \epsilon$ prescription in $-i \epsilon$.

When evaluated for real and positive external energies $\left.p_{h}^{0}=E_{h}, 21\right]$ gives

$$
\mathcal{M}_{a b}^{*}\left(E_{h}, \epsilon\right)=-\frac{\lambda^{V}}{S_{\#}} \prod_{i=1}^{L} \int_{-i \infty\left(\mathcal{C}^{*}\right)}^{+i \infty} \int_{\mathbb{R}^{3}} \frac{i d k_{i}^{0} d^{3} k_{i}}{(2 \pi)^{4}} \frac{1}{k_{i}^{2}-m^{2}-i \epsilon} \prod_{j=1}^{I-L} \frac{1}{q_{j}^{2}-m^{2}-i \epsilon} B\left(k_{i}, p_{h}\right),
$$

where we have inverted the orientation of $\mathcal{C}^{*}$ reabsorbing a minus sign, and used the fact that $\tilde{q}_{i}=q_{i}$ for real $p_{h}^{0}$. Therefore, Eq. 18 follows straightforwardly from 22 .

At that point we can show that the discontinuity of $\mathcal{M}$ in the rhs. of $(18)$ is given by the Cutkosky rules. Let us consider the case of two poles that converge in the limit $\epsilon \rightarrow 0$ at some real external energies $p_{h}^{0}=E_{h}$ corresponding to a specific Landau singularity. In Fig 1 , this situation is represented by the pole $\bar{k}_{2}^{0}(E)$, which approaches $\bar{k}_{6}^{0}$ and constrains the integration contour $\mathcal{C}$. Since $\sqrt{16}$ ) is of the same form of the integrals considered in [15], we can make use of the result of Cutkosky (skipping any useless rederivation of such result), which states that the discontinuity of $\mathcal{M}$ at the branch cut is given by the residue of the integrand in (16) at the pole, times a $(2 \pi i)$ numerical factor. We stress that only the propagators that go on shell at the Landau singularity under consideration contribute to 17). Therefore, 18 is obtained replacing in 16 each propagator that goes on shell at the singularity with a term $(-2 \pi i) \delta\left(p^{2}-m^{2}\right)$, exactly as in the case of a local scalar field theory. The only difference with respect to the local case is that one has to replace the integration region $\mathcal{C} \times \mathbb{R}^{3}$ with $\mathbb{R}^{4}$ for all the loop momenta contained in the on shell propagators. Indeed, the Cutkosky rules are still valid, mutatis mutandis, for the class of nonlocal theories under consideration.

We can make the derivation of the Cutkosky rules given above even more explicit, giving a procedure to evaluate the analytically continued amplitude (16) for generic diagrams. In order to proceed, we must determine how the integration contour $\left(\mathcal{I} \times \mathbb{R}^{3}\right)$ for the $i$-th loop variable $k_{i}$ is deformed into $\left(\mathcal{C}_{i} \times \mathbb{R}^{3}\right)$. In what follows, we will give a prescription for finding $\left(\mathcal{C}_{i} \times \mathbb{R}^{3}\right)$ proceeding iteratively. Since we will integrate $(16)$ one loop at time, it is useful to define how the deformed integration contour is obtained for a generic integral in one-loop variable. Let us consider the following integral:

$$
M\left(p_{h}\right)=\int_{\mathcal{I} \times \mathbb{R}^{3}} f\left(k, p_{h}\right) d k^{0} d^{3} k
$$

where $p_{h}$ represents the external momenta, and the function $f\left(k, p_{h}\right)$ has $m$ poles $\bar{k}_{l}^{0}\left(\vec{k}, p_{h}\right)$ with $l=1,2, \ldots m$ in the complex $k^{0}$ plane. We make the hypothesis that the poles $\bar{k}_{l}^{0}\left(\vec{k}, p_{h}\right)$ are not purely imaginary when the external energies $p_{h}^{0}$ are purely imaginary. Indeed, $\bar{k}_{l}^{0}\left(\vec{k}, p_{h}\right)$ are far from the integration contour for purely imaginary external energies, which is exactly the case of 14 . When we move $p_{h}^{0}$ to real positive values, the poles $\bar{k}_{l}^{0}\left(\vec{k}, p_{h}\right)$ will move in the $k^{0}$ complex plane, and some of them will pass through the integration contour $\mathcal{I}$ (see Fig II ). Therefore, the analytic continuation of the function $M\left(p_{h}\right)$ to real values of the external energies $p_{h}$ is obtained from (23) deforming the integration contour $\mathcal{I}$ around the poles that pinch the imaginary axis, i.e., 


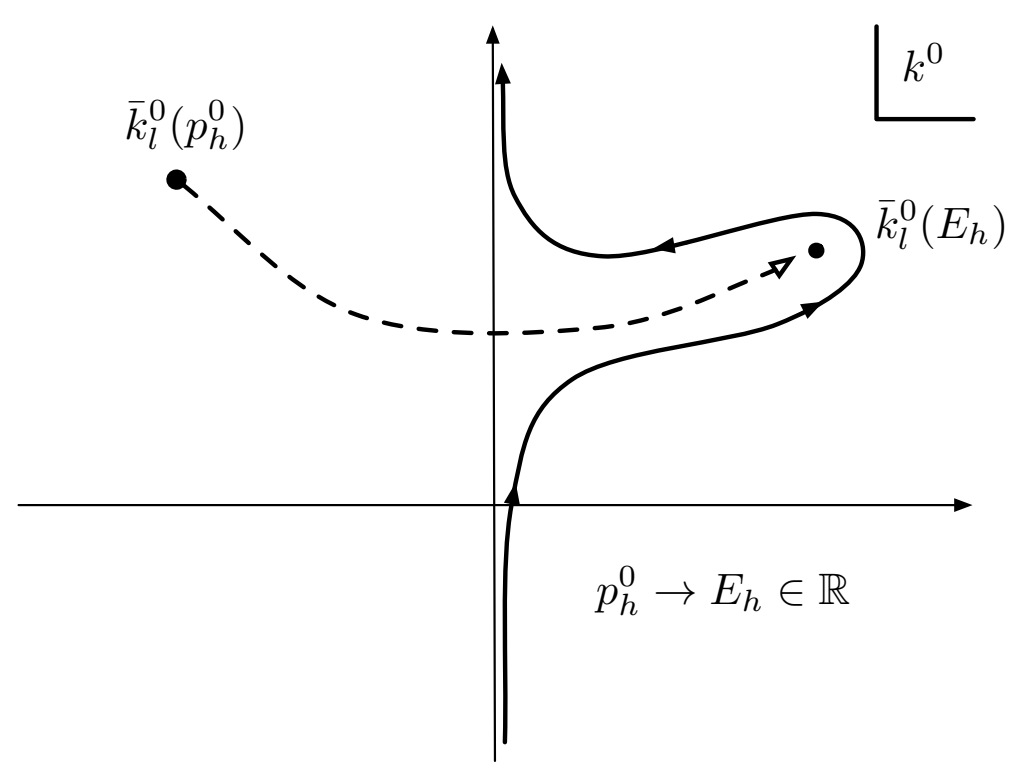

FIG. 2: The pole $\bar{k}_{l}^{0}\left(p_{h}\right)$ moves thorough the imaginary axes $\mathcal{I}$ when $p_{h}^{0} \rightarrow E_{h}^{0}$, and the integration contour $\mathcal{C}$ is obtained deforming $\mathcal{I}$ around $\bar{k}_{l}^{0}\left(E_{h}\right)$.

$$
M\left(p_{h}\right)=\int_{\mathcal{C} \times \mathbb{R}^{3}} f\left(k, p_{h}\right) d k^{0} d^{3} k
$$

If the integrand $f\left(k, p_{h}\right)$ is analytic, which will be always assumed hereafter, the integral on $\mathcal{C}$ equals the integral along the imaginary axis $\mathcal{I}$ plus the contributions of the residues of $f\left(k, p_{h}\right)$ at each pole that has passed through the imaginary axes in the limit $p_{h}^{0} \rightarrow E_{h} \in \mathbb{R}_{0}^{+}$, that is,

$$
M\left(p_{h}\right)=\int_{\mathcal{I} \times \mathbb{R}^{3}} f\left(k, p_{h}\right) d k^{0} d^{3} k \pm(2 \pi i) \int_{\mathbb{R}^{3}} \sum_{l} \operatorname{Res}\left\{f\left(k, p_{h}\right), \bar{k}_{l}^{0}\left(\vec{k}, p_{h}\right)\right\} d^{3} k .
$$

where the sum on the index $l$ in the second integral in (25) is limited to those poles that passed through the imaginary axes. The plus sign in 25) corresponds to poles that pass through $\mathcal{I}$ from left to right, while the minus sign corresponds to poles that move from right to left.

In order to evaluate the complex amplitude (14), we can write $\mathcal{M}$ as

$$
\mathcal{M}=-\frac{\lambda^{V}}{S_{\#}} \int_{\left(\mathcal{C}_{L} \times \mathbb{R}^{3}\right)} \frac{i d^{4} k_{L}}{(2 \pi)^{4}} \frac{F_{L}\left(k_{L}, p_{h}\right)}{k_{L}^{2}-m^{2}+i \epsilon}
$$

where we have defined

$$
F_{L}\left(k_{L}, p_{h}\right)=\int_{\left(\mathcal{C}_{L-1} \times \mathbb{R}^{3}\right)} \int_{\left(\mathcal{C}_{L-2} \times \mathbb{R}^{3}\right)} \ldots \int_{\left(\mathcal{C}_{1} \times \mathbb{R}^{3}\right)} \prod_{i=1}^{L-1} \frac{i d^{4} k_{i}}{(2 \pi)^{4}} \frac{1}{k_{i}^{2}-m^{2}+i \epsilon} \prod_{j=1}^{I-L} \frac{1}{q_{j}^{2}-m^{2}+i \epsilon} B\left(k_{i}, p_{h}\right),
$$

and proceeding iteratively, we define

$$
F_{L}\left(k_{L}, p_{h}\right)=\int_{\left(\mathcal{C}_{L-1} \times \mathbb{R}^{3}\right)} \frac{i d^{4} k_{L-1}}{(2 \pi)^{4}} \frac{F_{L-1}\left(k_{L}, k_{L-1}, p_{h}\right)}{k_{L-1}^{2}-m^{2}+i \epsilon},
$$

with

$$
F_{L-1}\left(k_{L}, k_{L-1}, p_{h}\right)=\int_{\left(\mathcal{C}_{L-2} \times \mathbb{R}^{3}\right)} \frac{i d^{4} k_{L-2}}{(2 \pi)^{4}} \frac{F_{L-2}\left(k_{L}, k_{L-1}, k_{L-2}, p_{h}\right)}{k_{L-2}^{2}-m^{2}+i \epsilon}
$$


and so on, until the last expressions for $F_{2}$ and $F_{1}$, namely

$$
\begin{aligned}
& F_{2}\left(k_{L}, k_{L-1}, \ldots, k_{2}, p_{h}\right)=\int_{\left(\mathcal{C}_{1} \times \mathbb{R}^{3}\right)} \frac{i d^{4} k_{1}}{(2 \pi)^{4}} \frac{F_{1}\left(k_{L}, k_{L-1}, \ldots, k_{1}, p_{h}\right)}{k_{1}^{2}-m^{2}+i \epsilon}, \\
& F_{1}\left(k_{L}, k_{L-1}, \ldots, k_{1}, p_{h}\right)=\prod_{j=1}^{I-L} \frac{1}{q_{j}^{2}-m^{2}+i \epsilon} B\left(k_{i}, p_{h}\right) .
\end{aligned}
$$

Equations 27$)-32$ can be recast by means of the recursive relation

$$
F_{i+1}\left(k_{L}, k_{L-1}, \ldots, k_{i+1}, p_{h}\right)=\int_{\left(\mathcal{C}_{i} \times \mathbb{R}^{3}\right)} \frac{i d^{4} k_{i}}{(2 \pi)^{4}} \frac{F_{i}\left(k_{L}, k_{L-1}, \ldots, k_{i}, p_{h}\right)}{k_{i}^{2}-m^{2}+i \epsilon} .
$$

Therefore, the complex amplitude $\mathcal{M}$ can be obtained evaluating the functions $F_{i}\left(k_{L}, \ldots, k_{i}, p_{h}\right)$ by means of the integration formula 25 starting from $F_{2}\left(k_{L}, k_{L-1}, \ldots, k_{2}\right)$ and then proceeding iteratively, i.e.,

$$
\begin{gathered}
F_{i+1}\left(k_{L}, \ldots, k_{i+1}, p_{h}\right)=\int_{\left(\mathcal{I} \times \mathbb{R}^{3}\right)} \frac{i d^{4} k_{i}}{(2 \pi)^{4}} \frac{F_{i}\left(k_{L}, \ldots, k_{i}, p_{h}\right)}{k_{i}^{2}-m^{2}+i \epsilon} \pm \int_{\left(\mathbb{R}^{3}\right)} \frac{i d^{3} k_{i}}{(2 \pi)^{4}}(2 \pi i) \sum_{l} \operatorname{Res}\left\{\frac{F_{i}\left(k_{L}, \ldots, k_{i}, p_{h}\right)}{k_{i}^{2}-m^{2}+i \epsilon}, \bar{k}_{i, l}^{0}\right\} \\
\mathcal{M}=-\frac{\lambda^{V}}{S_{\#}}\left[\int_{\left(\mathcal{I} \times \mathbb{R}^{3}\right)} \frac{i d^{4} k_{L}}{(2 \pi)^{4}} \frac{F_{L}\left(k_{L}, p_{h}\right)}{k_{L}^{2}-m^{2}+i \epsilon} \pm \int_{\left(\mathbb{R}^{3}\right)} \frac{i d^{3} k_{L}}{(2 \pi)^{4}}(2 \pi i) \sum_{l} \operatorname{Res}\left\{\frac{F_{L}\left(k_{L}, p_{h}\right)}{k_{L}^{2}-m^{2}+i \epsilon}, \bar{k}_{L, l}^{0}\right\}\right]
\end{gathered}
$$

where $\bar{k}_{i, l}^{0} \equiv \bar{k}_{i, l}^{0}\left(\vec{k}_{i}, p_{h}\right)$ are the poles of $F_{i}\left(k_{i}, p_{h}\right)$ in the $k_{i}^{0}$ complex plane, and the sum in $l$ is extended only to those poles passing through $\mathcal{I}$ in the limit $p_{h}^{0} \rightarrow E_{h} \in \mathbb{R}_{0}^{+}$.

We note that, in order to evaluate $F_{i+1}\left(k_{L}, k_{L-1}, \ldots, k_{i+1}, p_{h}\right)$ applying $(25)$, one must find the deformed contour $\mathcal{C}_{i} \times \mathbb{R}^{3}$ for the $i$-th loop integration variable. Indeed, one has to study the poles of the function $F_{i}\left(k_{L}, k_{L-1}, \ldots, k_{i}, p_{h}\right)$ and determine which ones pass through the imaginary axes of the complex plane of $k_{i}^{0}$ when we take the limit of real external energies, ${ }^{2}$ and this analysis must be repeated variable by variable. For this purpose it is useful to stress that, when the $i \epsilon$ factor is maintained, the poles of $F_{1}\left(k_{L}, k_{L-1}, \ldots, k_{1}, p_{h}\right)$ with positive imaginary part are well separated from those with negative imaginary part. Indeed, the deformed integration contour $\mathcal{C}_{i} \times \mathbb{R}^{3}$ cannot be pinched by the poles, and $\mathcal{M}$ is not singular. Therefore, the singularities and the corresponding branch cuts in $\mathcal{M}$ emerge in the limit $i \epsilon \rightarrow 0$.

Finally, having evaluated the complex amplitude by means of (34), the Cutkosky rules can be obtained evaluating $\mathcal{M}-\mathcal{M}^{*}$ in the limit $\epsilon \rightarrow 0$. Again, here we do not expect any substantial difference with respect to the case of a local field theory, since the propagators of local and nonlocal theories have the same poles.

We stress that this derivation of the Cutkosky rules is useful to enlighten the grounds of unitarity in nonlocal theories, but it is not necessary, since we have already shown that the amplitude (16) falls under the hypothesis of the Cutkosky theorem. Indeed, the discontinuity in its imaginary part is obtained applying the result of Cutkosky.

Before concluding this section, it is appropriate to comment briefly about the Lorentz invariance of the amplitude (16), since this point might be questioned by the reader. Let us consider an infinitesimal Lorentz transformation $\Lambda$. Applying this transformation to external and internal momenta we have

$$
\mathcal{M}^{\prime}=-\frac{\lambda^{V}}{S_{\#}} \int_{\left(\mathcal{C}_{L}^{\prime} \times \mathbb{R}^{3}\right)} \int_{\left(\mathcal{C}_{L-1}^{\prime} \times \mathbb{R}^{3}\right)} \ldots \int_{\left(\mathcal{C}_{1}^{\prime} \times \mathbb{R}^{3}\right)} \prod_{i=1}^{L} \frac{i d^{4} k_{i}}{(2 \pi)^{4}} \frac{1}{k_{i}^{2}-m^{2}+i \epsilon} \prod_{j=1}^{I-L} \frac{1}{q_{j}^{2}-m^{2}+i \epsilon} B\left(k_{i}, p_{h}\right),
$$

where we have used the fact that $B\left(k_{i}, p_{h}\right)$ depends on $k_{i}$ and $p_{h}$ through the square of their linear combinations, i.e., $k_{i}^{2}, p_{h}^{2}$ and $q_{i}^{2}$. Therefore, $B\left(k_{i}, p_{h}\right)$ is Lorentz invariant. Moreover, the contours $\mathcal{C}_{i}^{\prime}$ are obtained from $\mathcal{C}_{i}$ through the Lorentz transformation $\Lambda$. Since $\Lambda$ is infinitesimal, the two contours $\mathcal{C}_{i}^{\prime}$ and $\mathcal{C}$ will be infinitesimally close, and we can safely assume that there will be no poles among them. Indeed, since the integrand in (35) is analytic, we can replace the integration contours $\mathcal{C}_{i}^{\prime}$ with $\mathcal{C}$, obtaining $\mathcal{M}^{\prime}=\mathcal{M}$ for an infinitesimal Lorentz transformation. Therefore, being invariant under infinitesimal Lorentz transformations, the amplitude (16) will be invariant under finite Lorentz transformations.

\footnotetext{
${ }^{2}$ Note that the roots of $k_{i}^{2}-m^{2}+i \epsilon=0$ do not depend on $p_{h}$ an do not pinch the imaginary axis.
} 


\section{UNITARITY AND CUTKOSKY RULES}

In this section, we summarize briefly the relation between unitarity and Cutkosky rules (see [9, 10, 18, for a review). In particular, we will argue that, to complete the proof of the unitarity of nonlocal theories, we have to show that anomalous thresholds do not contribute to (17). This will be proved in Sec. IV.

The unitarity condition $S^{\dagger} S=1$ for the $S$ matrix can be expressed in terms of the $T$ matrix as $T-T^{\dagger}=i T^{\dagger} T$, where the $T$ matrix is defined by $S=1+i T$. Taking the expectation value of the unitarity condition between an initial incoming state $|a\rangle$ and an outgoing final state $\langle b|$ for a given process $a \rightarrow b$, one has

$$
T_{b a}-T_{a b}^{*}=i \sum_{c} T_{c b}^{*} T_{c a}
$$

Recasting the matrix elements of the $T$ matrix in terms of those of the invariant scattering amplitude $\mathcal{M}$ as

$T_{a b}=(2 \pi)^{4} \mathcal{M}_{a b} \delta^{(4)}\left(\sum_{i} p_{i}-\sum_{f} p_{f}\right)$, where $p_{i}$ and $p_{f}$ are the initial an final external momenta, the unitarity condition is finally expressed as

$$
\mathcal{M}_{b a}-\mathcal{M}_{a b}^{*}=i \sum_{c} \mathcal{M}_{c b}^{*} \mathcal{M}_{c a}(2 \pi)^{4} \delta^{(4)}\left(p_{c}-p_{a}\right),
$$

where $\mathcal{M}_{b a}=\langle b|\mathcal{M}| a\rangle$ is the sum of all the connected amputated diagrams for the process $a \rightarrow b$ (see [9, 10] for a review) and where we have neglected a global $\delta^{(4)}\left(\sum_{i} p_{i}-\sum_{f} p_{f}\right)$ multiplying both sides of Eq. (37). The sum in $c$ is made on all possible real (nonvirtual) intermediate states, i.e., on those states that give nonzero amplitudes $\mathcal{M}_{b c}=\langle b|\mathcal{M}| c\rangle$ and $\mathcal{M}_{a c}=\langle a|\mathcal{M}| c\rangle$. The interpretation of (37) is that at a Landau pole the amplitude $\mathcal{M}_{b a}$ has a branch cut singularity, which implies a discontinuity in its imaginary part above the corresponding threshold, which is related to the opening of a channel of production of intermediate real states $c$.

In order to prove the unitarity of the theory (3), we must show that (37) is satisfied for any process $a \rightarrow b$. We have already shown that the imaginary part of $\mathcal{M}$ at a specific Landau pole is given by the Cutkosky rules, replacing each propagator that goes on shell with a term $(-2 \pi i) \delta\left(q^{2}-m^{2}\right)$ and the integration contour $\mathcal{C} \times \mathbb{R}^{3}$ in the loops variables contained in $q$ with $\mathbb{R}^{4}$. The expression obtained in this way is usually referred as a cut diagram, since it is graphically represented by the same diagram as $\mathcal{M}$ in which the lines corresponding to the on shell propagators are cut (see 9, 10, 18, for review).

The Landau poles that are such that the corresponding cut diagram divide the original diagram in two are usually referred as normal thresholds. For such normal thresholds it is easy to show that (37) is verified, since this is a straightforward consequence of the Cutkosky rules (see [15] for review). Therefore, to complete our proof of the unitarity, we must show that only the Landau poles corresponding to normal thresholds contribute to the imaginary part of $\mathcal{M}$. This is necessary since some Landau poles are such that, cutting the on shell propagators, the diagram is not divided in two, and the imaginary part of the amplitude cannot be recast as in (37). Such Landau poles are usually referred as anomalous thresholds.

Even in the case of a local theory, proving that anomalous thresholds do not contribute to (37) is a difficult task, which requires methods based on coordinate space analysis of diagrams, as the largest time equation [18. However, the extension of such techniques to nonlocal theories is not obvious, because the largest time equation makes use of the fact that, in a local theory, the propagator can be divided in positive and negative energy parts, which is no longer the case in nonlocal theories. Yet, we can show that the diagrams that contribute to (37) in the nonlocal case are all and only those that contribute in the local case; indeed there is no contribution from diagrams corresponding to anomalous thresholds. In fact, we know that anomalous thresholds do not contribute in the local case, because the local theory is unitary.

Before proceeding to this proof in Sec. IV] it is instructive to compute the limit (17) for some simple diagrams that do not have anomalous thresholds, proving their unitarity. This will be done in Secs. IIIA and IIIB. There, we will calculate $\mathcal{M}_{b a}$ from (14) using the recursive equations (26 34). Then we will obtain $\mathcal{M}_{a b}^{*}\left(E_{h}, \epsilon\right)$ by complex conjugation, and finally we will calculate $\mathcal{M}_{a b}\left(E_{h}, \epsilon\right)-\mathcal{M}_{a b}^{*}\left(E_{h}, \epsilon\right)$. Of course, the result will be in agreement with the Cutkosky rules.

\section{A. One-loop diagram}

Let us consider the one-loop four-particle scattering diagram in Fig.3, for a nonlocal scalar field with interaction $\lambda \phi^{4} / 4$ !. The corresponding amputated amplitude in the Euclidean spacetime with purely imaginary external energies 


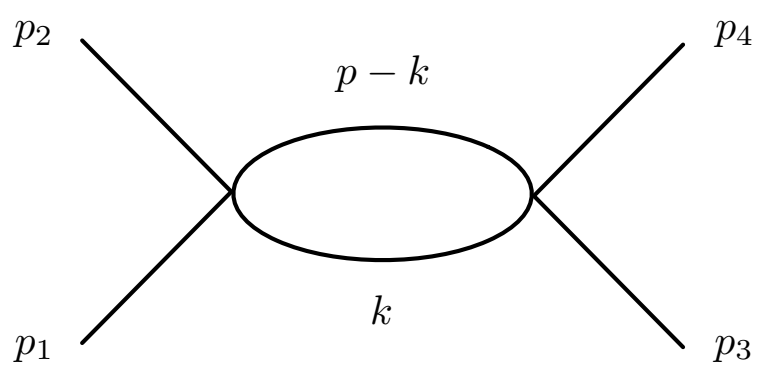

FIG. 3: Four-particle scattering amplitude in $\lambda \phi^{4} / 4$ ! theory at one-loop order.

is $^{3}$

$$
\mathcal{M}\left(p_{h}, \epsilon\right)=-\frac{\lambda^{2}}{2} \int_{\left(\mathcal{I} \times \mathbb{R}^{3}\right)} \frac{i d^{4} k}{(2 \pi)^{4}} \frac{\mathcal{V}\left(p_{1}, p_{2}, k, p-k\right)}{k^{2}-m^{2}+i \epsilon} \frac{\mathcal{V}\left(p-k, k, p_{3}, p_{4}\right)}{(k-p)^{2}-m^{2}+i \epsilon}=-\frac{\lambda^{2}}{2} \int_{\left(\mathcal{I} \times \mathbb{R}^{3}\right)} \frac{i d^{4} k}{(2 \pi)^{4}} \frac{F_{1}\left(k, p_{h}\right)}{k^{2}-m^{2}+i \epsilon}
$$

where, in agreement with the notations $26-31$,

$$
F_{1}\left(k, p_{h}\right) \equiv F_{1}\left(k, p_{1}, p_{2}, p_{3}, p_{4}\right)=\frac{B\left(k, p_{h}\right)}{(k-p)^{2}-m^{2}+i \epsilon},
$$

with $B\left(k, p_{h}\right) \equiv \mathcal{V}\left(p_{1}, p_{2}, k, p-k\right) \mathcal{V}\left(p-k, k, p_{3}, p_{4}\right)$, and $p=p_{1}+p_{2}=p_{3}+p_{4}$, where $p_{1}, p_{2}, p_{3}, p_{4}$ are the external momenta.

In order to continue analytically $\left(38\right.$ to real external energies, we should deform the integration contour $\left(\mathcal{I} \times \mathbb{R}^{3}\right)$ around the poles of the propagators in $(38)$ that pass through $\mathcal{I}$ when the energies $p_{h}^{0}$ become real, just as in 16 . Then, we can use the recursive procedure defined by (33) and (34), which make repeated use of the integration formula (25). Indeed, we must find the poles of the function $F_{1}\left(k, p_{h}\right)$ in the variable $k^{0}$ and determine how they move in the complex $k^{0}$ plane when the external energies are moved to their physical values $p_{1}^{0} \rightarrow E_{1} \in \mathbb{R}_{0}^{+}, p_{2}^{0} \rightarrow E_{2} \in \mathbb{R}_{0}^{+}$, $p_{3}^{0} \rightarrow E_{3} \in \mathbb{R}_{0}^{+}, p_{4}^{0} \rightarrow E_{4} \in \mathbb{R}_{0}^{+}$, so that $p^{0} \rightarrow E \in \mathbb{R}_{0}^{+}$.

The poles of the first propagator are

$$
\bar{k}_{1,2}^{0}= \pm \sqrt{\vec{k}^{2}+m^{2}-i \epsilon}
$$

Such poles do not depend on the external energies and remain always far from the imaginary axis. Indeed, $\bar{k}_{1,2}^{0}$ do not pinch $\mathcal{I}$ and do not contribute to the sum of residues in 25 . However, such poles overlap with those of the function $F_{1}\left(k, p_{h}\right)$ in the limit $i \epsilon \rightarrow 0$ for some values of the spatial loop momentum $\vec{k}$ and external momenta, constraining in between the deformed contour $\mathcal{C}$, that will be then pinched. That implies the occurrence of a singularity at some threshold energy and a corresponding branch cut discontinuity when $i \epsilon \rightarrow 0$.

The poles of $F_{1}\left(k, p_{h}\right)$ are given by the two zeros of its denominator, which are

$$
\bar{k}_{3,4}^{0}=p^{0} \pm \sqrt{(\vec{k}-\vec{p})^{2}+m^{2}-i \epsilon}
$$

These two poles are at the right and left of $p^{0}$, which is initially purely imaginary; indeed, $\bar{k}_{3}^{0}$ is at the right and $\bar{k}_{4}^{0}$ is at the left of the imaginary axis of the $k^{0}$ plane, for purely imaginary $p^{0}$. When $p^{0}$ is moved to its physical real and positive value $E$, the two poles $\bar{k}_{3,4}^{0}$ move to the right and it happens that the pole $\bar{k}_{4}^{0}$ passes through the imaginary axis for some values of the loop momenta $\vec{k}$ and the external energy (see Fig.4. Indeed, the integration contour $\mathcal{C}$ is obtained deforming the imaginary axis $\mathcal{I}$ around the pole $\bar{k}_{4}^{0}$ when such pole passes through $\mathcal{I}$, i.e., when $\Re\left\{\bar{k}_{4}^{0}\right\}>0$, where $\Re\{a\}$ is the real part of the complex number $a \in \mathbb{C}$.

Having defined the deformed integration contour, we can write the analytical continuation of the amplitude (38) as

\footnotetext{
${ }^{3}$ Hereafter, we omit the indices $a b$ in $\mathcal{M}_{a b}$.
} 


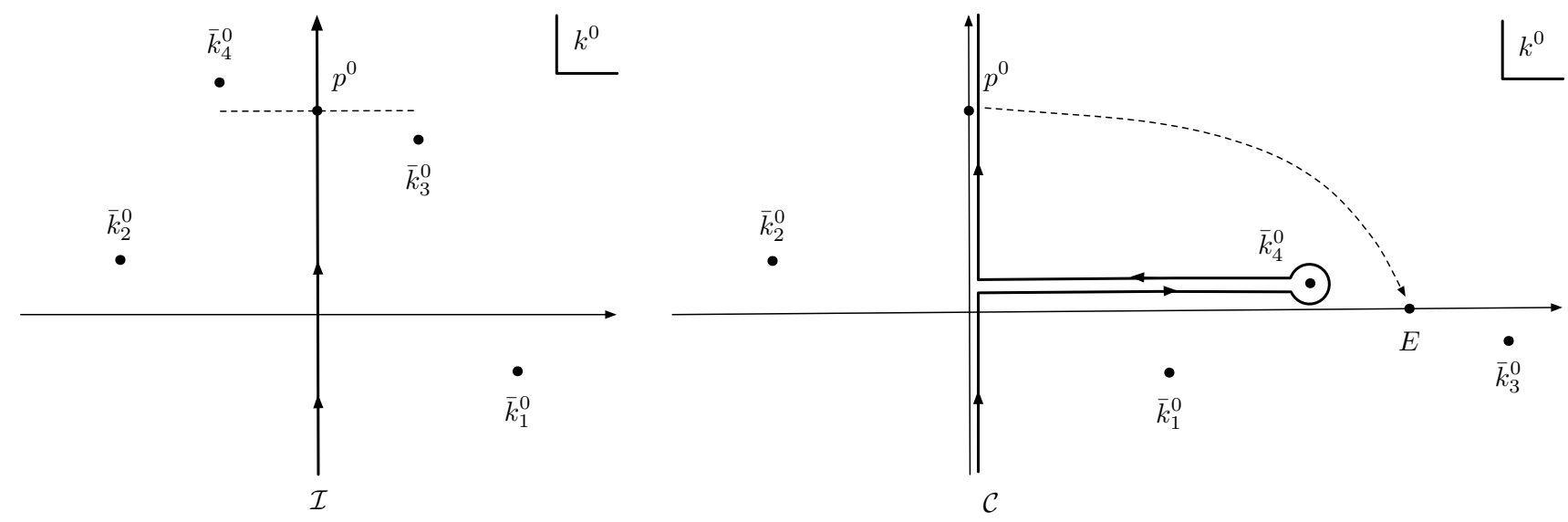

FIG. 4: (Left) We plot the poles $\bar{k}_{1}^{0}, \ldots, \bar{k}_{4}^{0}$ on the complex $k^{0}$ plane, when $p^{0}$ is purely imaginary. (Right) We plot the same poles for $p^{0}$ real and positive. Since $\bar{k}_{1}^{0}$ and $\bar{k}_{2}^{0}$ do not depend on $p^{0}$, their positions do not change when $p^{0} \rightarrow E$. On the contrary, $\bar{k}_{3}^{0}$ and $\bar{k}_{4}^{0}$ move to the right, and $\bar{k}_{4}^{0}$ passes through the imaginary axis $\mathcal{I}$ for some values of $\vec{k}$. We also plot the contour $\mathcal{C}$, which is obtained deforming $\mathcal{I}$ around $\bar{k}_{4}^{0}$.

$$
\mathcal{M}\left(p_{h}, \epsilon\right)=-\frac{\lambda^{2}}{2} \int_{\left(\mathcal{C} \times \mathbb{R}^{3}\right)} \frac{i d^{4} k}{(2 \pi)^{4}} \frac{1}{k^{2}-m^{2}+i \epsilon} \frac{B\left(k, p_{h}\right)}{(k-p)^{2}-m^{2}+i \epsilon}=-\frac{\lambda^{2}}{2} \int_{\left(\mathcal{C} \times \mathbb{R}^{3}\right)} \frac{i d^{4} k}{(2 \pi)^{4}} \frac{F_{1}\left(k, p_{h}\right)}{k^{2}-m^{2}+i \epsilon},
$$

where now $p^{0}=p_{1}^{0}+p_{2}^{0}=E \in \mathbb{R}_{0}^{+}$is real and positive.

According to 25 , we can divide this integral in two parts, separating the contribution of the residuals from the integral on $\mathcal{I}$, which gives

$$
\mathcal{M}\left(p_{h}, \epsilon\right)=-\frac{\lambda^{2}}{2}\left[\int_{\left(\mathcal{I} \times \mathbb{R}^{3}\right)} \frac{i d^{4} k}{(2 \pi)^{4}} \frac{F_{1}\left(k, p_{h}\right)}{k^{2}-m^{2}+i \epsilon}+\int_{\left(\mathbb{R}^{3}\right)} \frac{i d^{3} k}{(2 \pi)^{4}} 2 \pi i \sum_{l} \operatorname{Res}\left\{\frac{F_{1}\left(k, p_{h}\right)}{k^{2}-m^{2}+i \epsilon}, \bar{k}_{l}^{0}\right\}\right],
$$

where the sum is extended to all the residues $\bar{k}_{l}^{0}$ that pass through $\mathcal{I}$ when $p^{0}$ passes from purely imaginary to real and positive values. Therefore, the only pole that contributes to the sum of the residues in 43 is $\bar{k}_{4}^{0}=p^{0}-\sqrt{(\vec{k}-\vec{p})^{2}+m^{2}-i \epsilon}$, which passes from the left to the right of $\mathcal{I}$ when $p^{0} \rightarrow E$, for values of the external energies and internal loop momenta $\vec{k}$ such that $\Re\left\{\bar{k}_{4}^{0}\right\}>0$. Thus, Eq. 43 gives

$\mathcal{M}\left(p_{h}, \epsilon\right)=-\frac{\lambda^{2}}{2}\left[\int_{\left(\mathcal{I} \times \mathbb{R}^{3}\right)} \frac{i d^{4} k}{(2 \pi)^{4}} \frac{F_{1}(k)}{k^{2}-m^{2}+i \epsilon}+\left.\int_{\left(\mathbb{R}^{3}\right)} \frac{i d^{3} k}{(2 \pi)^{4}}(-2 \pi i)\left(\frac{\sigma\left(\Re\left\{k^{0}\right\}\right)}{2 \sqrt{(\vec{k}-\vec{p})^{2}+m^{2}-i \epsilon}} \frac{B\left(k, p_{h}\right)}{k^{2}-m^{2}+i \epsilon}\right)\right|_{k^{0}=\bar{k}_{4}^{0}}\right]$,

where

$$
\sigma(x)=\left\{\begin{array}{l}
1, \quad x>0 \\
1 / 2, \quad x=0 \\
0, \quad x<0
\end{array}\right.
$$

The $\sigma\left(\Re\left(\bar{k}_{4}^{0}\right)\right)$ in 25 implies that when the pole $\bar{k}_{4}^{0}$ is in the first or fourth quadrant of the complex plane it contributes a $2 \pi i$ times the residue. When it is on the imaginary axis $\mathcal{I}$, it contributes just the half, while when it is in the second and third quadrant it does not contribute. Moreover, when the pole is on the imaginary axis, the integral over $\mathcal{I}$ is intended as the principal part. Therefore, the support of $\sigma\left(\Re\left(\bar{k}_{4}^{0}\right)\right)$ defines the region of integration in $d^{3} k$. Furthermore, $\sigma\left(\Re\left(\bar{k}_{4}^{0}\right)\right)=1 / 2$ on a subset of $\mathbb{R}^{3}$ with zero measure; thus, such subset does not contribute to the second integral in 23 . 
In what follows, we will make use of the following relation:

$$
\left.\int_{\mathbb{R}^{3}} d^{3} k \frac{f(k, h)}{2 \sqrt{(\vec{k}-\vec{h})^{2}+m^{2}}}\right|_{k^{0} \equiv h^{0} \mp \sqrt{(\vec{k}-\vec{h})^{2}+m^{2}}}=\int_{\mathbb{R}^{4}} d^{4} k \sigma\left( \pm\left(h^{0}-k^{0}\right)\right) \delta\left((h-k)^{2}-m^{2}\right) f(k, h),
$$

where the $\sigma\left( \pm\left(h^{0}-k^{0}\right)\right)$ function selects the correct root $h^{0} \mp \sqrt{(\vec{k}-\vec{h})^{2}+m^{2}}$ of the delta function. Let us consider the last integral in 44 . Since $(\vec{k}-\vec{p})^{2}+m^{2} \neq 0$, we can neglect the $i \epsilon$ term in $\sqrt{(\vec{k}-\vec{p})^{2}+m^{2}-i \epsilon}$, since we are interested in the limit $\epsilon \rightarrow 0$ of the amplitude. Therefore, according to (46), in the limit of small $\epsilon$ one has

$$
\left.\int_{\left(\mathbb{R}^{3}\right)} d^{3} k\left(\frac{\sigma\left(\Re\left\{k^{0}\right\}\right)}{2 \sqrt{(\vec{k}-\vec{p})^{2}+m^{2}-i \epsilon}} \frac{B\left(k, p_{h}\right)}{k^{2}-m^{2}+i \epsilon}\right)\right|_{k^{0}=\bar{k}_{4}^{0}}=\int_{\left(\mathbb{R}^{4}\right)} d^{4} k \frac{B\left(k, p_{h}\right)}{k^{2}-m^{2}+i \epsilon} \sigma\left(k^{0}\right) \sigma\left(p^{0}-k^{0}\right) \delta\left((p-k)^{2}-m^{2}\right),(4
$$

where we have maintained the term $i \epsilon$ in the propagator $1 /\left(k^{2}-m^{2}+i \epsilon\right)$ since this propagator can go on shell. Therefore, the final expression of the analytic continuation (42) is obtained by replacing (47) in (44), obtaining

$$
\begin{aligned}
\mathcal{M}\left(p_{h}, \epsilon\right)=-\frac{\lambda^{2}}{2} & {\left[\int_{\left(\mathcal{I} \times \mathbb{R}^{3}\right)} \frac{i d^{4} k}{(2 \pi)^{4}} B\left(k, p_{h}\right) \frac{1}{k^{2}-m^{2}+i \epsilon} \frac{1}{(k-p)^{2}-m^{2}+i \epsilon}+\right.} \\
+ & \left.\int_{\left(\mathbb{R}^{4}\right)} \frac{i d^{4} k}{(2 \pi)^{4}} \frac{B\left(k, p_{h}\right)}{k^{2}-m^{2}+i \epsilon}(-2 \pi i) \sigma\left(k^{0}\right) \sigma\left(p^{0}-k^{0}\right) \delta\left((p-k)^{2}-m^{2}\right)\right] .
\end{aligned}
$$

We note that the first integral in $(48)$ is of the same kind of the integral as $(19)$; therefore, following the steps $(19)$ - $(22)$, one easily realizes that such integral is real in the limit $i \epsilon \rightarrow 0$, and it does not contribute to $\mathcal{M}^{-\mathcal{M}^{*}}$. Notice that $k^{2}-m^{2} \neq 0$ and $(k-p)^{2}-m^{2} \neq 0$ in the first integral of 48 because $k \in \mathcal{I} \times \mathbb{R}^{3}$ and $p \in \mathbb{R}_{0}^{+} \times \mathbb{R}^{3}$, so that the integrand is not singular on the integration contour.

We can now evaluate $\mathcal{M}\left(p_{h}, \epsilon\right)-\mathcal{M}\left(p_{h}, \epsilon\right)^{*}$ in the limit $i \epsilon \rightarrow 0$ and verify the validity of the Cutkosky rules for our nonlocal theory. We have

$$
\begin{aligned}
& \mathcal{M}\left(p_{h}, \epsilon\right)-\mathcal{M}\left(p_{h}, \epsilon\right)^{*}= \\
& \quad=-\frac{\lambda^{2}}{2} \int_{\left(\mathbb{R}^{4}\right)} \frac{i d^{4} k}{(2 \pi)^{4}} B\left(k, p_{h}\right)(-2 \pi i) \sigma\left(k^{0}\right) \sigma\left(p^{0}-k^{0}\right) \delta\left((p-k)^{2}-m^{2}\right)\left[\frac{1}{k^{2}-m^{2}+i \epsilon}-\frac{1}{k^{2}-m^{2}-i \epsilon}\right] .
\end{aligned}
$$

In order to recast the second integral of 49 , we just note that it is performed on the real $\mathbb{R}^{4}$ space; thus, one is allowed to use the formula

$$
\lim _{\epsilon \rightarrow 0}\left\{\frac{1}{k^{2}-m^{2}+i \epsilon}-\frac{1}{k^{2}-m^{2}-i \epsilon}\right\}=(-2 \pi i) \delta\left(k^{2}-m^{2}\right),
$$

obtaining

$$
\lim _{\epsilon \rightarrow 0}\left\{\mathcal{M}\left(p_{h}, \epsilon\right)-\mathcal{M}\left(p_{h}, \epsilon\right)^{*}\right\}=-\frac{\lambda^{2}}{2} \int_{\left(\mathbb{R}^{4}\right)} \frac{i d^{4} k}{(2 \pi)^{4}} B\left(k, p_{h}\right)(-2 \pi i)^{2} \sigma\left(p^{0}-k^{0}\right) \delta\left((p-k)^{2}-m^{2}\right) \sigma\left(k^{0}\right) \delta\left(k^{2}-m^{2}\right)
$$

where we have used the fact that $k^{0} \in \mathbb{R}$ when $i \epsilon \rightarrow 0$, which implies that $\sigma\left(\Re\left\{k^{0}\right\}\right)=\sigma\left(k^{0}\right)$. The $\sigma\left(k^{0}\right)$ in 51 , selects the positive $k^{0}$ root of the delta functions, corresponding to the physical energy of real intermediate states when the loop momenta are on shell.

Equation (51) is just the nonlocal version of the standard local Cutkosky rules, which state that the discontinuity of the amplitude at some cut is given by replacing each propagator

$$
\frac{i}{p^{2}-m^{2}+i \epsilon} \quad \longrightarrow \quad(-2 \pi i) \sigma\left(p^{0}\right) \delta\left(p^{2}-m^{2}+i \epsilon\right) .
$$

The only difference is that, in the case of a nonlocal theory, one has also to replace the integration contour $\mathcal{C} \times \mathbb{R}^{3}$ with $\mathbb{R}^{4}$. 


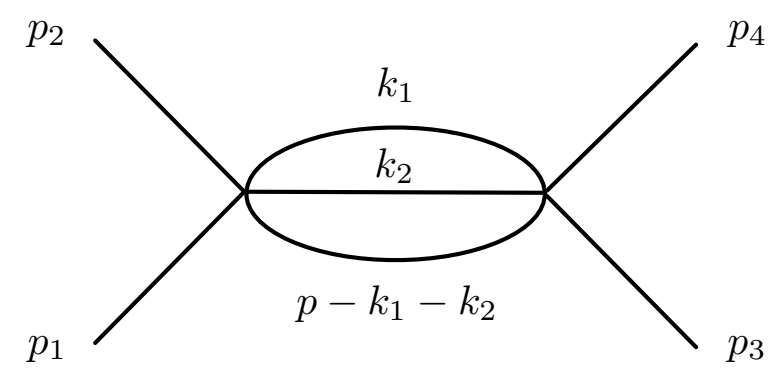

FIG. 5: Four-particle scattering amplitude at two loops in $\lambda \phi^{5}$ theory.

Finally, we note that, in all the theories we are interested in, the vertex function is such that $\mathcal{V}=1$ when the corresponding momenta are on shell. For instance, that happens when the function $H(z)$ in $(13)$ is such that $H\left(p^{2}=m^{2}\right)=0$. In such theories, one has $B\left(k, p_{h}\right)=1$ when all the momenta are on shell, and 53 simplifies

$$
\mathcal{M}\left(p_{h}, \epsilon\right)-\mathcal{M}\left(p_{h}, \epsilon\right)^{*}=-\frac{\lambda^{2}}{2} \int_{\left(\mathbb{R}^{4}\right)} \frac{i d^{4} k}{(2 \pi)^{4}}(-2 \pi i) \sigma\left(k^{0}\right) \delta\left((k-p)^{2}-m^{2}\right)(-2 \pi i) \sigma\left(p^{0}\right) \delta\left(k^{2}-m^{2}\right) .
$$

For these special but very common [1, 2, 4, 6, 12, 13] theories we can replace 52 , with

$$
\frac{i e^{-H\left(p^{2}\right)}}{p^{2}-m^{2}+i \epsilon} \quad \longrightarrow \quad(-2 \pi i) \sigma\left(p^{0}\right) \delta\left(p^{2}-m^{2}+i \epsilon\right) .
$$

\section{B. Two-loop diagram}

In this section, we study the two-loop diagram in Fig 5 for an Euclidean scalar field theory with $\lambda \phi^{5} / 5$ ! interaction. The complex amplitude for this process is

$$
\begin{aligned}
\mathcal{M}\left(p_{h}, \epsilon\right) & =-\frac{\lambda^{2}}{2} \int_{\left(\mathcal{I} \times \mathbb{R}^{3}\right)^{2}} \frac{i d^{4} k_{1}}{(2 \pi)^{4}} \frac{i d^{4} k_{2}}{(2 \pi)^{4}} \frac{1}{k_{1}^{2}-m^{2}+i \epsilon} \frac{\mathcal{V}\left(p, k_{1}, k_{2}, p-k_{1}-k_{2}\right)}{k_{2}^{2}-m^{2}+i \epsilon} \frac{\mathcal{V}\left(k_{1}, k_{2}, p-k_{1}-k_{2}, p\right)}{\left(k_{1}+k_{2}-p\right)^{2}-m^{2}+i \epsilon} \\
& =-\frac{\lambda^{2}}{2} \int_{\left(\mathcal{I} \times \mathbb{R}^{3}\right)} \frac{i d^{4} k_{2}}{(2 \pi)^{4}} \frac{\tilde{F}_{2}\left(k_{2}, p_{h}\right)}{k_{2}^{2}-m^{2}+i \epsilon},
\end{aligned}
$$

where we are assuming purely imaginary external energies $p_{1}^{0}, p_{2}^{0}, p_{3}^{0}, p_{4}^{0}$, and where we introduced the definitions

$$
\begin{aligned}
& \tilde{F}_{2}\left(k_{2}, p_{h}\right)=\int_{\left(\mathcal{I} \times \mathbb{R}^{3}\right)} \frac{i d^{4} k_{1}}{(2 \pi)^{4}} \frac{1}{k_{1}^{2}-m^{2}+i \epsilon} \frac{\tilde{B}\left(k_{1}, k_{2}, p_{h}\right)}{\left(k_{1}+k_{2}-p\right)^{2}-m^{2}+i \epsilon}=\int_{\left(\mathcal{I} \times \mathbb{R}^{3}\right)} \frac{i d^{4} k_{1}}{(2 \pi)^{4}} \frac{\tilde{F}_{1}\left(k_{2}, k_{1}, p_{h}\right)}{k_{1}^{2}-m^{2}+i \epsilon}, \\
& \tilde{F}_{1}\left(k_{2}, k_{1}, p_{h}\right)=\frac{\tilde{B}\left(k_{1}, k_{2}, p_{h}\right)}{\left(k_{1}+k_{2}-p\right)^{2}-m^{2}+i \epsilon},
\end{aligned}
$$

with

$$
\tilde{B}\left(k_{1}, k_{2}, p_{h}\right) \equiv \mathcal{V}\left(p, k_{1}, k_{2}, p-k_{1}-k_{2}\right) \mathcal{V}\left(k_{1}, k_{2}, p-k_{1}-k_{2}, p\right),
$$

and $p=p_{1}+p_{2}=p_{3}+p_{4}$ as the external momentum.

The expressions (55) and (56) are well defined when the external energies are purely imaginary because in that case the poles of the propagators are far from the integration contour $\left(\mathcal{I} \times \mathbb{R}^{3}\right)^{2}$. Therefore, to find the physical amplitude and prove the Cutkosky rules, we have to continue analytically $(55)$ and $(56)$ to real and positive external energies. This is done deforming the integration contour around the poles that pass through $\left(\mathcal{I} \times \mathbb{R}^{3}\right)^{2}$ when $p_{1}^{0} \rightarrow E_{1} \in \mathbb{R}_{0}^{+}$, $p_{2}^{0} \rightarrow E_{2} \in \mathbb{R}_{0}^{+}, p_{3}^{0} \rightarrow E_{3} \in \mathbb{R}_{0}^{+}$, and $p_{4}^{0} \rightarrow E_{4} \in \mathbb{R}_{0}^{+}$. The physical amplitude becomes

$$
\mathcal{M}\left(p_{h}, \epsilon\right)=-\frac{\lambda^{2}}{2} \int_{\left(\mathcal{C}_{2} \times \mathbb{R}^{3}\right)} \frac{i d^{4} k_{2}}{(2 \pi)^{4}} \frac{\tilde{F}_{2}\left(k_{2}, p_{h}\right)}{k_{2}^{2}-m^{2}+i \epsilon},
$$




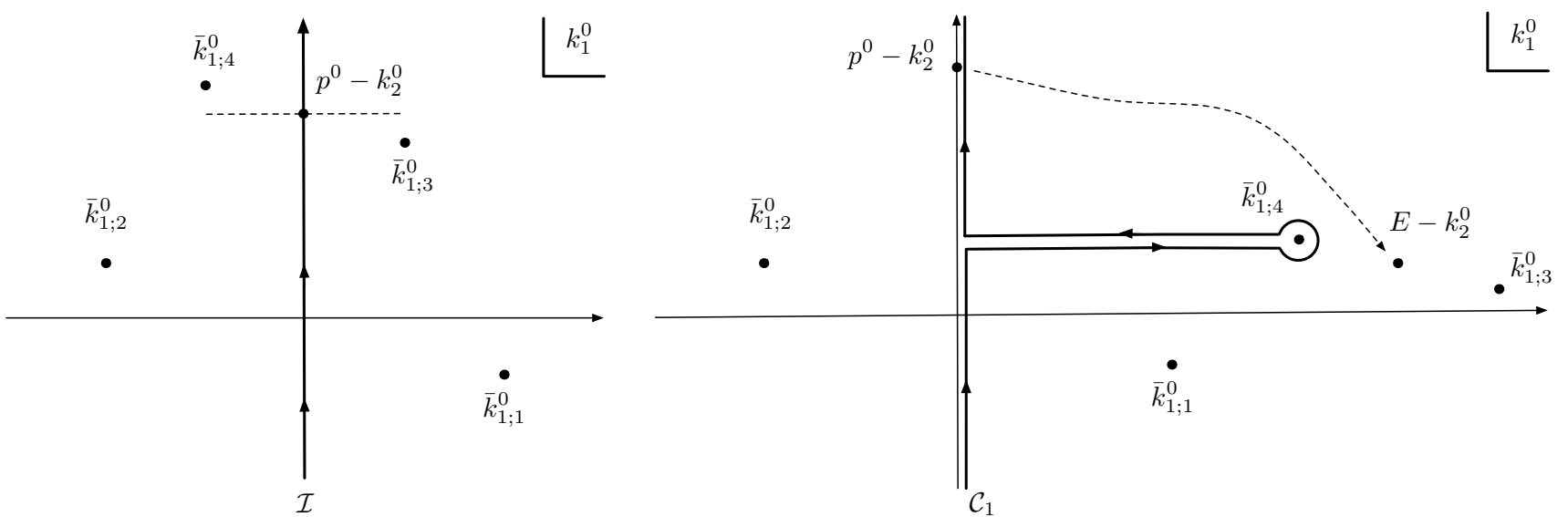

FIG. 6: (Left) We plot the poles $\bar{k}_{1 ; 1}^{0}, \ldots, \bar{k}_{1 ; 4}^{0}$ on the complex $k_{1}^{0}$ plane, when $p^{0}$ is purely imaginary, which implies that $k_{2}^{0}$ is purely imaginary too. (Right) We plot the same poles for $p^{0}$ real and positive. Since $\bar{k}_{1 ; 1}^{0}$ and $\bar{k}_{1 ; 2}^{0}$ do not depend on $p^{0}$, their positions do not change when $p^{0} \rightarrow E$. On the contrary, $\bar{k}_{1 ; 3}^{0}$ and $\bar{k}_{1 ; 4}^{0}$ move to the right, and $\bar{k}_{4}^{0}$ passes through the imaginary axis $\mathcal{I}$ for some values of $\vec{k}_{1}$. We also plot the contour $\mathcal{C}_{1}$, which is obtained deforming $\mathcal{I}$ around $\bar{k}_{1 ; 4}^{0}$.

with

$$
\tilde{F}_{2}\left(k_{2}, p_{h}\right)=\int_{\left(\mathcal{C}_{1} \times \mathbb{R}^{3}\right)} \frac{i d^{4} k_{1}}{(2 \pi)^{4}} \frac{1}{k_{1}^{2}-m^{2}+i \epsilon} \frac{\tilde{B}\left(k_{1}, k_{2}, p\right)}{\left(k_{1}-\left(p-k_{2}\right)\right)^{2}-m^{2}+i \epsilon}=\int_{\left(\mathcal{C}_{1} \times \mathbb{R}^{3}\right)} \frac{i d^{4} k_{1}}{(2 \pi)^{4}} \frac{\tilde{F}_{1}\left(k_{2}, k_{1}, p_{h}\right)}{k_{1}^{2}-m^{2}+i \epsilon},
$$

where the contours $\left(\mathcal{C}_{1} \times \mathbb{R}^{3}\right)$ and $\left(\mathcal{C}_{2} \times \mathbb{R}^{3}\right)$ are determined by the analysis of the poles of $\tilde{F}_{1}\left(k_{2}, k_{1}, p_{h}\right)$ and $\tilde{F}_{2}\left(k_{2}, p_{h}\right)$ as follows.

Let us start from the expression of $\tilde{F}_{2}\left(k_{2}, p_{h}\right)$ in $(59)$. In order to apply the integral formula 25$)$, we have to find the integration contour $\left(\mathcal{C}_{1} \times \mathbb{R}^{3}\right)$ by determining which one of the poles of $\tilde{F}_{1}\left(k_{2}, k_{1}, p_{h}\right)$ moves through the imaginary axis of the $k_{1}^{0}$ plane when $p^{0} \rightarrow E \in \mathbb{R}_{0}^{+}$. To proceed, we can use the analogy with the one-loop case as a guideline. In fact, (59) is the same as 42 with the replacements

$$
k \rightarrow k_{1}, \quad p \rightarrow p-k_{2}, \quad F_{1}(k, p) \rightarrow \tilde{F}_{1}\left(k_{1}, p-k_{2}\right), \quad B\left(k, p_{h}\right) \rightarrow \tilde{B}\left(k_{1}, k_{2}, p_{h}\right) .
$$

The only difference concerns the positions of the poles in the $k_{1}^{0}$ complex plane, which now also depend on $k_{2}$. The roots of $k_{1}^{2}-m^{2}+i \epsilon=0$, i.e.,

$$
\bar{k}_{1 ; 1,2}^{0}= \pm \sqrt{\vec{k}_{1}^{2}+m^{2}-i \epsilon}
$$

do not depend on $p^{0}$ and they are always far from the imaginary axes. The poles of $\tilde{F}_{2}\left(k_{2}, p_{h}\right)$ are

$$
\bar{k}_{1 ; 3,4}^{0}=p^{0}-k_{2}^{0} \pm \sqrt{\left(\vec{k}_{1}+\vec{k}_{2}-\vec{p}\right)^{2}+m^{2}-i \epsilon},
$$

and they are at the left and right of the imaginary axes when $p^{0}$ is purely imaginary, because in that case also $k_{2}^{0}$ is purely imaginary, since we integrate it on $\mathcal{I}$ [see 55 ] . Both the poles $\bar{k}_{1 ; 3,4}^{0}$ move to the right when $p^{0} \rightarrow E \in \mathbb{R}_{0}^{+}$, so that the only pole that might cross the imaginary axes is

$$
\bar{k}_{1 ; 4}^{0}=p^{0}-k_{2}^{0}-\sqrt{\left(\vec{p}-\vec{k}_{1}-\vec{k}_{2}\right)^{2}+m^{2}-i \epsilon},
$$

and this happens only when $\Re\left\{\bar{k}_{1 ; 4}^{0}\right\}>0$, see Fig. Therefore, $\left(\mathcal{C}_{1} \times \mathbb{R}^{3}\right)$ is obtained deforming $\left(\mathcal{I} \times \mathbb{R}^{3}\right)$ around $\bar{k}_{1 ; 4}^{0}$ when it crosses the imaginary axes. Now using 44 we can directly derive the expression of $\tilde{F}_{2}\left(k_{2}, p_{h}\right)$ as

$$
\begin{aligned}
\tilde{F}_{2}\left(k_{2}, p_{h}\right) & =\int_{\left(\mathcal{I} \times \mathbb{R}^{3}\right)} \frac{i d^{4} k_{1}}{(2 \pi)^{4}} \frac{1}{k_{1}^{2}-m^{2}+i \epsilon} \frac{\tilde{B}\left(k_{1}, k_{2}, p\right)}{\left(k_{1}+k_{2}-p\right)^{2}-m^{2}+i \epsilon} \\
& +\left.\int_{\left(\mathbb{R}^{3}\right)} \frac{i d^{3} k_{1}}{(2 \pi)^{4}}(-2 \pi i)\left(\frac{\sigma\left(\Re\left\{k_{1}^{0}\right\}\right)}{2 \sqrt{\left(\vec{p}-\vec{k}_{1}-\vec{k}_{2}\right)^{2}+m^{2}-i \epsilon}} \frac{B\left(k, p_{h}\right)}{k_{1}^{2}-m^{2}+i \epsilon}\right)\right|_{k_{1}^{0}=\bar{k}_{1 ; 4}^{0}},
\end{aligned}
$$


Therefore, (58) becomes

$$
\begin{aligned}
\mathcal{M}\left(p_{h}, \epsilon\right)=- & \frac{\lambda^{2}}{2} \int_{\left(\mathcal{C}_{2} \times \mathbb{R}^{3}\right)} \frac{i d^{4} k_{2}}{(2 \pi)^{4}} \frac{1}{k_{2}^{2}-m^{2}+i \epsilon}\left\{\int_{\left(\mathcal{I} \times \mathbb{R}^{3}\right)} \frac{i d^{4} k_{1}}{(2 \pi)^{4}} \frac{1}{k_{1}^{2}-m^{2}+i \epsilon} \frac{\tilde{B}\left(k_{1}, k_{2}, p\right)}{\left(k_{1}+k_{2}-p\right)^{2}-m^{2}+i \epsilon}\right. \\
& \left.+\left.\int_{\left(\mathbb{R}^{3}\right)} \frac{i d^{3} k_{1}}{(2 \pi)^{4}}(-2 \pi i)\left(\frac{\sigma\left(\Re\left\{k_{1}^{0}\right\}\right)}{2 \sqrt{\left(\vec{p}-\vec{k}_{1}-\vec{k}_{2}\right)^{2}+m^{2}-i \epsilon}} \frac{B\left(k, p_{h}\right)}{k_{1}^{2}-m^{2}+i \epsilon}\right)\right|_{k_{1}^{0}=\bar{k}_{1 ; 4}^{0}}\right\} .
\end{aligned}
$$

We stress that the delta function in 65 has to be interpreted according to 46 when $k_{2}^{0} \in I$ is purely imaginary. Now we have to analyze the poles of the integrand in $(65)$ in order to find contour $\left(\mathcal{C}_{2} \times \mathbb{R}^{3}\right)$. For the first integral in 65), we have

$$
\begin{aligned}
& \int_{\left(\mathcal{C}_{2} \times \mathbb{R}^{3}\right)} \frac{i d^{4} k_{2}}{(2 \pi)^{4}} \frac{1}{k_{2}^{2}-m^{2}+i \epsilon} \int_{\left(\mathcal{I} \times \mathbb{R}^{3}\right)} \frac{i d^{4} k_{1}}{(2 \pi)^{4}} \frac{1}{k_{1}^{2}-m^{2}+i \epsilon} \frac{\tilde{B}\left(k_{1}, k_{2}, p\right)}{\left(k_{1}+k_{2}-p\right)^{2}-m^{2}+i \epsilon} \\
& =\int_{\left(\mathcal{I} \times \mathbb{R}^{3}\right)} \frac{i d^{4} k_{1}}{(2 \pi)^{4}} \frac{1}{k_{1}^{2}-m^{2}+i \epsilon} \int_{\left(\mathcal{C}_{2} \times \mathbb{R}^{3}\right)} \frac{i d^{4} k_{2}}{(2 \pi)^{4}} \frac{1}{k_{2}^{2}-m^{2}+i \epsilon} \frac{\tilde{B}\left(k_{1}, k_{2}, p\right)}{\left(k_{1}+k_{2}-p\right)^{2}-m^{2}+i \epsilon} \\
& =\int_{\left(\mathcal{I} \times \mathbb{R}^{3}\right)} \frac{i d^{4} k_{1}}{(2 \pi)^{4}} \frac{1}{k_{1}^{2}-m^{2}+i \epsilon}\left[\int_{\left(\mathcal{I} \times \mathbb{R}^{3}\right)} \frac{i d^{4} k_{2}}{(2 \pi)^{4}} \frac{1}{k_{2}^{2}-m^{2}+i \epsilon} \frac{\tilde{B}\left(k_{1}, k_{2}, p\right)}{\left(k_{1}+k_{2}-p\right)^{2}-m^{2}+i \epsilon}\right. \\
& \left.+\left.\int_{\left(\mathbb{R}^{3}\right)} \frac{i d^{3} k_{2}}{(2 \pi)^{4}}(-2 \pi i)\left(\frac{\sigma\left(\Re\left\{k_{2}^{0}\right\}\right)}{2 \sqrt{\left(\vec{p}-\vec{k}_{1}-\vec{k}_{2}\right)^{2}+m^{2}-i \epsilon}}\right)\right|_{k_{2}^{0}=\bar{k}_{2 ; 4}^{0}}\right],
\end{aligned}
$$

where in the first equality we have just inverted the order of integration, while the integral in $d^{4} k_{2}$ has been obtained using (64) with the replacement $k_{1} \leftrightarrow k_{2}$, with the definition

$$
\bar{k}_{2 ; 4}^{0}=p^{0}-k_{1}^{0}-\sqrt{\left(\vec{p}-\vec{k}_{1}-\vec{k}_{2}\right)^{2}+m^{2}-i \epsilon} .
$$

We note that the two integrals in (66) are real in the limit $\epsilon \rightarrow 0$, therefore, they will not contribute to $\mathcal{M}-\mathcal{M}^{*}$. In fact, for what concerns the first integral in (66), one can easily repeat the steps $(19)-(22)$ and conclude that (66) is real for $\epsilon \rightarrow 0$. For the second integral in 66 , we note that $k_{1} \in \mathcal{I} \times \mathbb{R}^{3}, k_{2}^{0}=\bar{k}_{2 ; 4}^{0}$ and $\vec{k}_{2} \in \mathbb{R}^{3}$, so that $k_{1}^{2}-m^{2} \in \mathbb{R}$ while $k_{2}^{2}-m^{2} \in \mathbb{R}$ when $\epsilon \rightarrow 0$, and therefore such integral is real for $\epsilon \rightarrow 0$.

Let us now consider the second integral in 65 . We have to evaluate this integral according to the integration formula (25), that is,

$$
\int_{\left(\mathcal{C}_{2} \times \mathbb{R}^{3}\right)} \frac{i d^{4} k_{2}}{(2 \pi)^{4}} \frac{G\left(k_{2}, p_{h}\right)}{k_{2}^{2}-m^{2}+i \epsilon}=\int_{\left(\mathcal{I} \times \mathbb{R}^{3}\right)} \frac{i d^{4} k_{2}}{(2 \pi)^{4}} \frac{G\left(k_{2}, p_{h}\right)}{k_{2}^{2}-m^{2}+i \epsilon}+\int_{(\mathbb{R})^{3}} \frac{i d^{3} k_{2}}{(2 \pi)^{4}}(2 \pi i) \sum_{h} \operatorname{Res}\left\{\frac{G\left(k_{2}, p_{h}\right)}{k_{2}^{2}-m^{2}+i \epsilon}, \bar{k}_{2 ; h}^{0}\right\},
$$

where we have defined

$$
\begin{aligned}
G\left(k_{2}, p_{h}\right) & =\left.\int_{\left(\mathbb{R}^{3}\right)} \frac{i d^{3} k_{1}}{(2 \pi)^{4}}\left(\frac{(-2 \pi i) \sigma\left(\Re\left\{k_{1}^{0}\right\}\right)}{2 \sqrt{\left(\vec{k}_{1}+\vec{k}_{2}-\vec{p}\right)^{2}+m^{2}-i \epsilon}} \frac{\tilde{B}\left(k_{1}, k_{2}, p\right)}{k_{1}^{2}-m^{2}+i \epsilon}\right)\right|_{k_{1}^{0}=\bar{k}_{1 ; 4}^{0}} \\
& =\left.\int_{\left(\mathbb{R}^{3}\right)} \frac{i d^{3} k_{1}}{(2 \pi)^{4}}\left(\frac{(-2 \pi i)}{2 \sqrt{\left(\vec{k}_{1}+\vec{k}_{2}-\vec{p}\right)^{2}+m^{2}-i \epsilon}} \frac{\sigma\left(\Re\left\{k_{1}^{0}\right\}\right)}{k_{1}^{0}+\sqrt{{\overrightarrow{k_{1}}}^{2}+m^{2}-i \epsilon}} \frac{\tilde{B}\left(k_{1}, k_{2}, p\right)}{k_{1}^{0}-\sqrt{{\overrightarrow{k_{1}}}^{2}+m^{2}-i \epsilon}}\right)\right|_{k_{1}^{0}=\bar{k}_{1 ; 4}^{0}} .
\end{aligned}
$$

The sum on the rhs of $(68)$ is limited to the residues corresponding to the poles $\bar{k}_{2 ; h}^{0}$ that pass through the imaginary axis of the $k_{2}^{0}$ plane when we take real positive external energies, i.e., $p^{0} \rightarrow E \in \mathbb{R}_{0}^{+}$, and the contour $\left(\mathcal{C}_{2} \times \mathbb{R}^{3}\right)$ in 68 is obtained deforming $\left(\mathcal{I} \times \mathbb{R}^{3}\right)$ around the same poles.

Therefore, to compute 68 we have to find the poles of the function $\left.G\left(k_{2}, p_{h}\right)\right) /\left(k_{2}^{2}-m^{2}+i \epsilon\right)$ and study their dependence on $p^{0}$. The first two poles are given by the zeros of the denominator and they are located in

$$
\bar{k}_{2 ; 1,2}^{0}= \pm \sqrt{\vec{k}_{2}^{2}+m^{2}-i \epsilon} .
$$



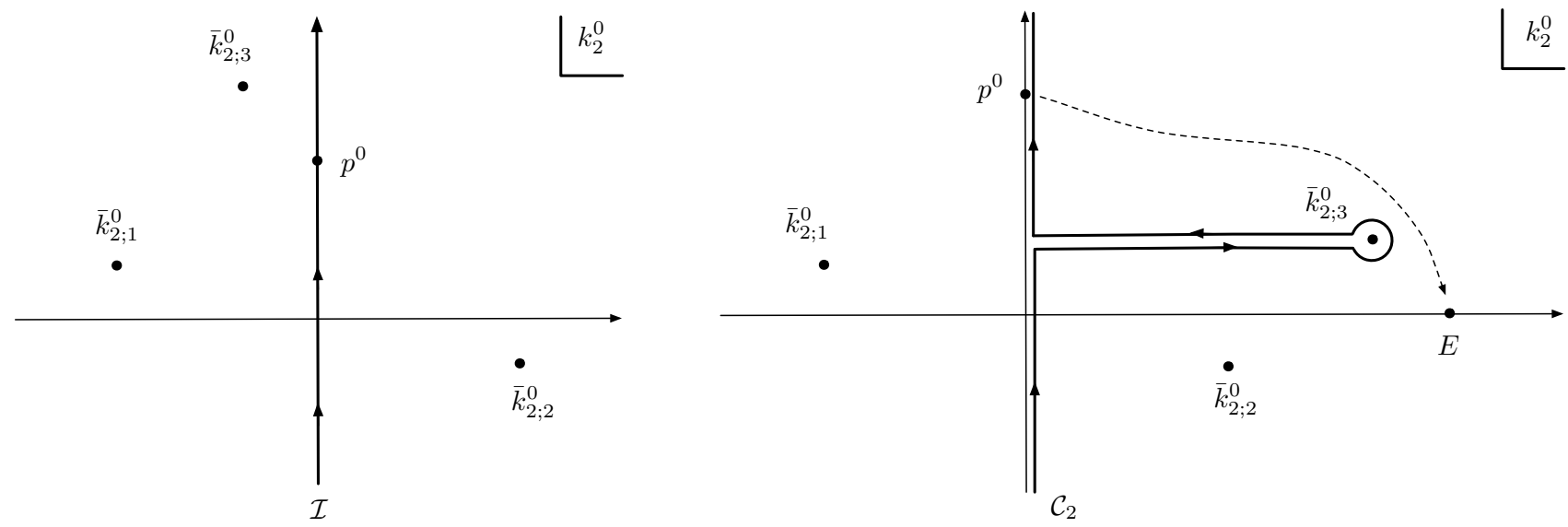

FIG. 7: (Left) We plot the poles $\bar{k}_{2 ; 1}^{0}, \bar{k}_{2 ; 2}^{0}, \bar{k}_{2 ; 3}^{0}$ on the complex $k_{2}^{0}$ plane, when $p^{0}$ is purely imaginary. (Right) We plot the same poles for $p^{0}$ real and positive. Since $\bar{k}_{2 ; 1}^{0}$ and $\bar{k}_{2 ; 2}^{0}$ do not depend on $p^{0}$, their positions do not change when $p^{0} \rightarrow E$. On the contrary, $\bar{k}_{2 ; 3}^{0}$ move to the right, and it passes through the imaginary axis $\mathcal{I}$ for some values of $\vec{k}_{2}$. We also plot the contour $\mathcal{C}_{2}$, which is obtained deforming $\mathcal{I}$ around $\bar{k}_{2 ; 3}^{0}$.

Such poles are in the second and fourth quadrant of the $k_{2}$ complex plane, far away from the imaginary axes $\mathcal{I}$. Moreover, $\bar{k}_{2 ; 1,2}^{0}$ never cross $\mathcal{I}$ because they do not depend on $p^{0}$. Therefore, they do not contribute to the sum of residues in (68). It remains to find the poles of $G\left(k_{2}, p_{h}\right)$. Due to the factor $\sigma\left(\Re\left\{k_{1}^{0}\right\}\right)$, which implies the positivity of $\bar{k}_{1 ; 4}^{0}$, only the last denominator in 69 can be null, then $G\left(k_{2}, p_{h}\right)$ has only one pole at

$$
\bar{k}_{1 ; 4}^{0}=\sqrt{{\overrightarrow{k_{1}}}^{2}+m^{2}-i \epsilon}
$$

so that such pole is found solving 71 for $\bar{k}_{2}^{0}$, which gives

$$
\bar{k}_{2 ; 3}^{0}=p^{0}-\sqrt{{\overrightarrow{k_{1}}}^{2}+m^{2}-i \epsilon}-\sqrt{\left(\vec{k}_{1}+\vec{k}_{2}-\vec{p}\right)^{2}+m^{2}-i \epsilon} .
$$

When the external energy $p^{0}$ is taken to be purely imaginary as it is in 55 , the poles $\bar{k}_{1 ; 4}^{0}$ and $\bar{k}_{2 ; 3}^{0}$ are on the left of the imaginary axis of the $k_{1}^{0}$ and $k_{2}^{0}$ complex planes, respectively (see Figs. 6 and 7). However, when $p^{0} \rightarrow E \in \mathbb{R}_{0}^{+}$ both $\bar{k}_{1 ; 4}^{0}$ and $\bar{k}_{2 ; 3}^{0}$ pass through such imaginary axis and their real parts become simultaneously positive for

$$
E>\Re\left\{\sqrt{{\overrightarrow{k_{1}}}^{2}+m^{2}-i \epsilon}+\sqrt{\left(\vec{k}_{1}+\vec{k}_{2}-\vec{p}\right)^{2}+m^{2}-i \epsilon}\right\},
$$

(see Fig.s 6 and 7). Therefore, $\bar{k}_{2 ; 3}^{0}$ contributes to the sum in 68 only when 73 is verified, and in that case $\Re\left\{\bar{k}_{2 ; 3}^{0}\right\}>0$. Therefore, the residue of $G\left(k_{2}, p_{h}\right) /\left(k_{2}^{2}-m^{2}+i \epsilon\right)$ at the pole $\bar{k}_{2 ; 3}^{0}$ is:

$\operatorname{Res}\left\{\frac{G\left(k_{2}, p_{h}\right)}{\left(k_{2}^{2}-m^{2}+i \epsilon\right)}, \bar{k}_{2 ; 3}^{0}\right\}=\left.\int_{\left(\mathbb{R}^{3}\right)} \frac{i d^{3} k_{1}}{(2 \pi)^{4}}\left(\frac{1}{k_{2}^{2}-m^{2}+i \epsilon} \frac{(-2 \pi i) \sigma\left(\Re\left\{k_{1}^{0}\right\}\right)}{2 \sqrt{\left(\vec{p}-\vec{k}_{1}-\vec{k}_{2}\right)^{2}+m^{2}-i \epsilon}} \frac{-\tilde{B}\left(k_{1}, k_{2}, p_{h}\right)}{2 \sqrt{\vec{k}_{1}^{2}+m^{2}-i \epsilon}}\right)\right|_{k_{1}^{0}=\bar{k}_{1 ; 4}^{0}} ^{k_{2}^{0}=\bar{k}_{2 ; 3}^{0}}$.

This expression can be used to evaluate $(68)$, which reads

$\int_{\left(\mathcal{C}_{2} \times \mathbb{R}^{3}\right)} \frac{i d^{4} k_{2}}{(2 \pi)^{4}} \frac{G\left(k_{2}, p_{h}\right)}{k_{2}^{2}-m^{2}+i \epsilon}=\int_{\left(\mathcal{I} \times \mathbb{R}^{3}\right)} \frac{i d^{4} k_{2}}{(2 \pi)^{4}} \frac{G\left(k_{2}, p_{h}\right)}{k_{2}^{2}-m^{2}+i \epsilon}+\int_{(\mathbb{R})^{3}} \frac{i d^{3} k_{2}}{(2 \pi)^{4}}(2 \pi i) \sigma\left(\Re\left\{\bar{k}_{2 ; 3}^{0}\right\}\right) \operatorname{Res}\left\{\frac{G\left(k_{2}, p_{h}\right)}{k_{2}^{2}-m^{2}+i \epsilon}, \bar{k}_{2 ; h}^{0}\right\}$,

where we have used the fact that $\bar{k}_{2 ; h}^{0}$ is the only pole contributing to the residues and we have introduced the term $\sigma\left(\Re\left\{\bar{k}_{2 ; 3}^{0}\right\}\right)$ since we know that the residue at $\bar{k}_{2 ; h}^{0}$ contributes only for $\bar{k}_{2 ; h}^{0}>0$. 
Since we are interested in the limit $\epsilon \rightarrow 0$ of the amplitude, we can neglect the $i \epsilon$ term in $\sqrt{\left(\vec{p}-\vec{k}_{1}-\vec{k}_{2}\right)^{2}+m^{2}-i \epsilon}$ and $\sqrt{\left(\vec{p}-\vec{k}_{1}-\vec{k}_{2}\right)^{2}+m^{2}-i \epsilon}$, while maintaining it in the propagator $k_{2}^{2}-m^{2}+i \epsilon$, so that in the small $\epsilon$ limit we have

$$
\begin{aligned}
& \int_{(\mathbb{R})^{3}} \frac{i d^{3} k_{2}}{(2 \pi)^{4}}(2 \pi i) \sigma\left(\Re\left\{\bar{k}_{2 ; 3}^{0}\right\}\right) \operatorname{Res}\left\{\frac{G\left(k_{2}, p_{h}\right)}{k_{2}^{2}-m^{2}+i \epsilon}, \bar{k}_{2 ; h}^{0}\right\}= \\
& =\left.\int_{(\mathbb{R})^{3}} \frac{i d^{3} k_{2}}{(2 \pi)^{4}}(2 \pi i) \sigma\left(\Re\left\{\bar{k}_{2 ; 3}^{0}\right\}\right) \int_{\left(\mathbb{R}^{3}\right)} \frac{i d^{3} k_{1}}{(2 \pi)^{4}}\left(\frac{1}{k_{2}^{2}-m^{2}+i \epsilon} \frac{(-2 \pi i) \sigma\left(\Re\left\{k_{1}^{0}\right\}\right)}{2 \sqrt{m^{2}+\left(\vec{k}_{1}+\vec{k}_{2}-\vec{p}\right)^{2}}} \frac{-\tilde{B}\left(k_{1}, k_{2}, p_{h}\right)}{2 \sqrt{m^{2}+\vec{k}_{1}^{2}-i \epsilon}}\right)\right|_{k_{1}^{0}=\bar{k}_{1 ; 4}^{0}} ^{k_{2}^{0}=\bar{k}_{2 ; 3}^{0}} \\
& =\int_{(\mathbb{R})^{4}} \frac{i d^{4} k_{2}}{(2 \pi)^{4}} \int_{\left(\mathbb{R}^{4}\right)} \frac{i d^{4} k_{1}}{(2 \pi)^{4}} \frac{(-2 \pi i)^{2} \sigma\left(k_{1}^{0}\right) \sigma\left(k_{2}^{0}\right) \sigma\left(p^{0}-k_{1}^{0}-k_{2}^{0}\right) \delta\left(\left(p-k_{1}-k_{2}\right)^{2}-m^{2}\right) \delta\left(k_{1}^{2}-m^{2}\right) \tilde{B}\left(k_{1}, k_{2}, p_{h}\right)}{k_{2}^{2}-m^{2}+i \epsilon},
\end{aligned}
$$

where the last equality is obtained applying (46) repeatedly. Using $(69)$ and $(74)-(75)$ we have

$$
\begin{aligned}
& \int_{\left(\mathcal{C} \times \mathbb{R}^{3}\right)} \frac{i d^{4} k_{2}}{(2 \pi)^{4}} \frac{G\left(k_{2}, p_{h}\right)}{k_{2}^{2}-m^{2}+i \epsilon} \\
& =\left.\int_{\left(\mathcal{I} \times \mathbb{R}^{3}\right)} \frac{i d^{4} k_{2}}{(2 \pi)^{4}} \frac{1}{k_{2}^{2}-m^{2}+i \epsilon} \int_{\left(\mathbb{R}^{3}\right)} \frac{i d^{3} k_{1}}{(2 \pi)^{4}}\left(\frac{(-2 \pi i) \sigma\left(\Re\left\{k_{1}^{0}\right\}\right)}{2 \sqrt{\left(\vec{k}_{1}+\vec{k}_{2}-\vec{p}\right)^{2}+m^{2}-i \epsilon}} \frac{\tilde{B}\left(k_{1}, k_{2}, p\right)}{k_{1}^{2}-m^{2}+i \epsilon}\right)\right|_{k_{1}^{0}=\bar{k}_{1 ; 4}^{0}} \\
& +\int_{\left(\mathbb{R}^{4}\right)} \frac{i d^{4} k_{2}}{(2 \pi)^{4}} \int_{\left(\mathbb{R}^{4}\right)} \frac{i d^{4} k_{1}}{(2 \pi)^{4}} \frac{(-2 \pi i)^{2} \sigma\left(k_{1}^{0}\right) \sigma\left(k_{2}^{0}\right) \sigma\left(p^{0}-k_{1}^{0}-k_{2}^{0}\right) \delta\left(\left(p-k_{1}-k_{2}\right)^{2}-m^{2}\right) \delta\left(k_{1}^{2}-m^{2}\right) \tilde{B}\left(k_{1}, k_{2}, p\right)}{k_{2}^{2}-m^{2}+i \epsilon} .
\end{aligned}
$$

The first integral in 77 is real in the limit $\epsilon \rightarrow 0$, since $k_{2} \in \mathcal{I} \times \mathbb{R}^{3}, k_{1}^{0}=\bar{k}_{1 ; 4}^{0}, \vec{k}_{1} \in \mathbb{R}^{3}$, thus $k_{2}^{2}-m^{2} \in \mathbb{R}$ while $k_{1}^{2}-m^{2} \in \mathbb{R}$ for $\epsilon \rightarrow 0$, so that it will not contribute to $\mathcal{M}-\mathcal{M}^{*}$ in such limit.

Finally, the analytic continuation (56) to real external energies of the complex amplitude (55) is obtained substituting (66) and (77) in (65), obtaining

$$
\begin{aligned}
& \mathcal{M}\left(p_{h}, \epsilon\right)=-\frac{\lambda^{2}}{2}\left[\int_{\left(\mathcal{I} \times \mathbb{R}^{3}\right)} \frac{i d^{4} k_{1}}{(2 \pi)^{4}} \frac{1}{k_{1}^{2}-m^{2}+i \epsilon} \int_{\left(\mathcal{I} \times \mathbb{R}^{3}\right)} \frac{i d^{4} k_{2}}{(2 \pi)^{4}} \frac{1}{k_{2}^{2}-m^{2}+i \epsilon} \frac{\tilde{B}\left(k_{1}, k_{2}, p\right)}{\left(k_{1}+k_{2}-p\right)^{2}-m^{2}+i \epsilon}\right. \\
& \quad+\left.\int_{\left(\mathcal{I} \times \mathbb{R}^{3}\right)} \frac{i d^{4} k_{1}}{(2 \pi)^{4}} \frac{1}{k_{1}^{2}-m^{2}+i \epsilon} \int_{\left(\mathbb{R}^{3}\right)} \frac{i d^{3} k_{2}}{(2 \pi)^{4}}(-2 \pi i)\left(\frac{\sigma\left(\Re\left\{k_{2}^{0}\right\}\right)}{2 \sqrt{\left(\vec{p}-\vec{k}_{1}-\vec{k}_{2}\right)^{2}+m^{2}-i \epsilon}}\right)\right|_{k_{2}^{0}=\bar{k}_{2 ; 4}^{0}} \\
& \quad+\left.\int_{\left(\mathcal{I} \times \mathbb{R}^{3}\right)} \frac{i d^{4} k_{2}}{(2 \pi)^{4}} \frac{1}{k_{2}^{2}-m^{2}+i \epsilon} \int_{\left(\mathbb{R}^{3}\right)} \frac{i d^{3} k_{1}}{(2 \pi)^{4}}\left(\frac{(-2 \pi i) \sigma\left(\Re\left\{k_{1}^{0}\right\}\right)}{2 \sqrt{\left(\vec{k}_{1}+\vec{k}_{2}-\vec{p}\right)^{2}+m^{2}-i \epsilon}} \frac{\tilde{B}\left(k_{1}, k_{2}, p\right)}{k_{1}^{2}-m^{2}+i \epsilon}\right)\right|_{k_{1}^{0}=\bar{k}_{1 ; 4}^{0}} \\
& \left.\quad+\int_{\left(\mathbb{R}^{4}\right)} \frac{i d^{4} k_{2}}{(2 \pi)^{4}} \int_{\left(\mathbb{R}^{4}\right)} \frac{i d^{4} k_{1}}{(2 \pi)^{4}} \frac{(-2 \pi i)^{2} \sigma\left(k_{1}^{0}\right) \sigma\left(k_{2}^{0}\right) \sigma\left(p^{0}-k_{1}^{0}-k_{2}^{0}\right) \delta\left(\left(p-k_{1}-k_{2}\right)^{2}-m^{2}\right) \delta\left(k_{1}^{2}-m^{2}\right) \tilde{B}\left(k_{1}, k_{2}, p\right)}{k_{2}^{2}-m^{2}+i \epsilon}\right],
\end{aligned}
$$

Therefore, we can evaluate the limit $\epsilon \rightarrow 0$ of the imaginary part of the amplitude. We have already shown that the only term contributing is given by the last integral in $(78)$, since all the other terms are real. Therefore we have

$$
\begin{aligned}
\mathcal{M}\left(p_{h}, \epsilon\right)-\mathcal{M}\left(p_{h}, \epsilon\right)^{*}=- & \frac{\lambda^{2}}{2} \int_{\left(\mathbb{R}^{4}\right)} \frac{i d^{4} k_{1}}{(2 \pi)^{4}} \int_{\left(\mathbb{R}^{4}\right)} \frac{i d^{4} k_{2}}{(2 \pi)^{4}}(-2 \pi i)^{2} \delta\left(\left(p-k_{1}-k_{2}\right)^{2}-m^{2}\right) \delta\left(k_{1}^{2}-m^{2}\right) \times \\
& \tilde{B}\left(k_{1}, k_{2}, p\right) \sigma\left(k_{1}^{0}\right) \sigma\left(k_{2}^{0}\right) \sigma\left(p^{0}-k_{1}^{0}-k_{2}^{0}\right)\left[\frac{1}{k_{2}^{2}-m^{2}+i \epsilon}-\frac{1}{k_{2}^{2}-m^{2}-i \epsilon}\right],
\end{aligned}
$$

and using $(50)$ we have

$$
\begin{array}{r}
\lim _{\epsilon \rightarrow 0} \mathcal{M}\left(p_{h}, \epsilon\right)-\mathcal{M}\left(p_{h}, \epsilon\right)^{*}=-\frac{\lambda^{2}}{2} \int_{\left(\mathbb{R}^{4}\right)} \frac{i d^{4} k_{1}}{(2 \pi)^{4}} \int_{\left(\mathbb{R}^{4}\right)} \frac{i d^{4} k_{2}}{(2 \pi)^{4}}(-2 \pi i) \sigma\left(p^{0}-k_{1}^{0}-k_{2}^{0}\right) \delta\left(\left(p-k_{1}-k_{2}\right)^{2}-m^{2}\right) \\
\times(-2 \pi i) \sigma\left(k_{1}^{0}\right) \delta\left(k_{1}^{2}-m^{2}\right)(-2 \pi i) \sigma\left(k_{2}^{0}\right) \delta\left(k_{2}^{2}-m^{2}\right) \tilde{B}\left(k_{1}, k_{2}, p\right),
\end{array}
$$


which confirms the validity of the Cutkosky rules for the two-loop process in Fig 5 Finally, in the special case in which the function $H(z)$ in $\sqrt{13}$ is such that $H\left(p^{2}=m^{2}\right)=0$, one has $\tilde{B}\left(k_{1}, k_{2}, p\right)=1$ when the internal momenta are on shell. Therefore, one has

$$
\begin{aligned}
& \lim _{\epsilon \rightarrow 0} \mathcal{M}\left(E_{h}, \epsilon\right)-\mathcal{M}\left(E_{h}, \epsilon\right)^{*}= \\
& =-\frac{\lambda^{2}}{2} \int_{\left(\mathbb{R}^{4}\right)} \frac{i d^{4} k_{1}}{(2 \pi)^{4}} \int_{\left(\mathbb{R}^{4}\right)} \frac{i d^{4} k_{2}}{(2 \pi)^{4}}(-2 \pi i)^{3} \sigma\left(p^{0}-k_{1}^{0}-k_{2}^{0}\right) \delta\left(\left(p-k_{1}-k_{2}\right)^{2}-m^{2}\right) \sigma\left(k_{1}^{0}\right) \delta\left(k_{1}^{2}-m^{2}\right) \sigma\left(k_{2}^{0}\right) \delta\left(k_{2}^{2}-m^{2}\right) .
\end{aligned}
$$

\section{UNITARITY OF THE THEORY}

In this section, we will complete the proof of the unitarity of the nonlocal theory (3), showing that the imaginary part of the complex amplitudes receive contributions only from Landau poles that correspond to normal thresholds, so that the unitary condition (37) is fulfilled.

In the previous sections, we have shown how to compute the imaginary part of a scattering amplitude. Stating from (16) or $\sqrt{19}$ and applying repeatedly the residual formula $\sqrt{25}$ according to $(26,34)$, one obtains the discontinuity in the imaginary part of the scattering amplitude as a sum of terms

$$
\begin{aligned}
\lim _{\epsilon \rightarrow 0} \mathcal{M}\left(E_{h}, \epsilon\right) & -\mathcal{M}\left(E_{h}, \epsilon\right)^{*}= \\
= & -\frac{\lambda^{V}}{S_{\#}} \sum \int_{\Omega_{1}} \ldots \int_{\Omega_{L}} \prod_{i=1}^{L} \frac{i d^{4} k_{i}}{(2 \pi)^{4}} \prod_{k=1}^{N}(-2 \pi i) \delta\left(Q_{k}^{2}-m^{2}\right) \sigma\left(Q_{k}^{0}\right) \prod_{j=1}^{I-N} \frac{1}{Q_{j}^{2}-m^{2}+i \epsilon} B\left(k_{i}, p_{h}\right),
\end{aligned}
$$

where the $Q_{k}$ are the momenta corresponding to internal lines. Each term in the sum in 82 corresponds to a cut diagram in which $N$ propagators are on shell while $I-N$ are not on shell. Moreover, for each term, the $i$-th integration region $\Omega_{i}$ can be $\mathbb{R}^{4}$ or $\mathcal{I} \times \mathbb{R}^{3}$, depending whether the corresponding momenta $k_{i}$ is contained in the propagator of one of the cut lines or not.

Let us consider the local version of the action (3), obtained assuming unitary form factor, i.e., $H(-\sigma \square) \equiv 0$, that is,

$$
\mathcal{L}_{\phi}=\frac{1}{2} \partial_{\mu} \varphi \partial^{\mu} \varphi-\frac{1}{2} m^{2} \varphi^{2}-\lambda \sum_{n=4}^{N} \frac{c_{n}}{n !} \varphi^{n}
$$

Considering a generic amplitude $\mathcal{M}$, it is easy to show that, if a cut diagram does not contribute to $\mathcal{M}^{-} \mathcal{M}^{*}$ in the case of the local theory (83), then the corresponding cut diagram in the nonlocal theory (3) does not contribute to (82). The proof is obtained noting that for the local theory 83 one has

$$
\lim _{\epsilon \rightarrow 0} \mathcal{M}\left(E_{h}, \epsilon\right)-\mathcal{M}\left(E_{h}, \epsilon\right)^{*}=-\frac{\lambda^{V}}{S_{\#}} \sum \int_{\Omega_{1}} \ldots \int_{\Omega_{L}} \prod_{i=1}^{L} \frac{i d^{4} k_{i}}{(2 \pi)^{4}} \prod_{k=1}^{N}(-2 \pi i) \delta\left(Q_{k}^{2}-m^{2}\right) \sigma\left(Q_{k}^{0}\right) \prod_{j=1}^{I-N} \frac{1}{Q_{j}^{2}-m^{2}+i \epsilon}
$$

and (84) contains the same terms with the same delta functions in 82 , with the only difference being that $B$ is replaced with one. This is due to the fact that $B\left(k_{i}, p_{h}\right) \neq 0$ by hypothesis; indeed' the nonlocality does not change the pole structure of the complex amplitudes, as discussed in Sec. II. Since the delta functions in 82 and (84) comes from the residues at the poles, it follows that each term in 82 corresponds to a term in (84) with the same delta functions.

Let us assume that the contribution of a specific cut diagram in (84) is zero, i.e.,

$$
\int_{\Omega_{1}} \ldots \int_{\Omega_{L}} \prod_{i=1}^{L} \frac{i d^{4} k_{i}}{(2 \pi)^{4}} \prod_{k=1}^{N}(-2 \pi i) \delta\left(Q_{k}^{2}-m^{2}\right) \sigma\left(Q_{k}^{0}\right) \prod_{j=1}^{I-N} \frac{1}{Q_{j}^{2}-m^{2}+i \epsilon}=0
$$

When this happens, this quantity is null due to the fact that $\prod_{k=1}^{N} \delta\left(Q_{k}^{2}-m^{2}\right) \sigma\left(Q_{k}^{0}\right)=0$, which implies that also the corresponding term in 82 is zero because it contains the same delta functions.

Therefore, if a cut diagram does not contribute to (84), it does not contribute to (82). Since the local theory (83) is unitary, which can be demonstrated making use of the largest time equation [18, the sum (84) does not contain 


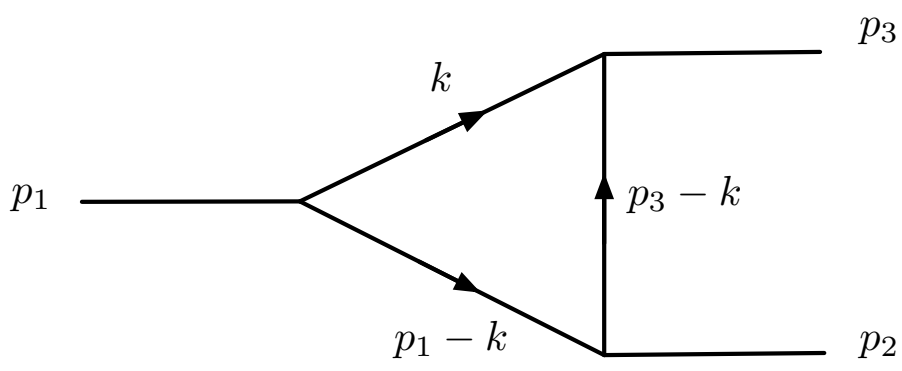

FIG. 8: Triangle diagram. The kinematics allows for the three internal lines to go on shell at the same time. This gives a cut diagram that divides the triangle diagram in three parts when it is cut along the three on shell propagators. Such cut diagram corresponds to an anomalous threshold.

contributions from cut diagrams corresponding to anomalous thresholds; indeed, the same will be true for 822 in the case of our nonlocal theory. Furthermore, the unitarity of (83) also implies that (84) contains contributions from all the cut diagrams that appear on the rhs of (37); indeed' the same will be true in the case of the nonlocal theory (3). This, together with the Cutkosky rules, proves the unitarity of the nonlocal field theory (3).

We conclude this section noting that (82) contains cut diagrams with at most $L+1$ cut lines, where $L$ is the number of loops in the diagram. This is easily understood if we think about how the delta functions arise. In fact, for each integration in (16), one can apply (25) obtaining a residue coming from the a pole of the integrand plus a term with no residue. Since each residue gives a delta function, in (16) we have terms containing at most the product of $L$ delta functions. Indeed, 82 contains terms encompassing at most the product of $L+1$ delta functions, the last delta coming from the difference between the propagators, according to $(50)$. For instance, in the one loop diagram studied in Sec. III A the only contribution to the imaginary part of the amplitudes comes from a diagram with two cut lines, while in the two-loop example of Sec. IIIB, one has only a cut diagram containing three delta. This property of 82 . is very useful, since it makes possible to exclude immediately the contribution of cut diagrams containing more than $L+1$ cut lines.

In what follows we will clarify what we have demonstrated above by means of two examples corresponding to the triangle and box diagrams, which do have anomalous thresholds.

\section{A. Triangle diagram}

Let us consider the triangle diagrams in Fig 8 . When the masses of the internal lines are chosen properly, the kinematics allows for the three internal lines to go on shell at the same time for some values of the external momenta. This situation corresponds to an anomalous threshold since, cutting the lines corresponding to the three on shell propagators, the diagram is not divided in two parts. In such situation the question arises whether this anomalous threshold contributes to the lhs of (37), since if this would be the case, such contribution could not be expressed as a product $i \mathcal{M}_{c b}^{*} \mathcal{M}_{c a}$ for some intermediate state $c$, and the theory would not be unitary.

Here we show that this is not the case, and (82) does not contain contributions from such anomalous threshold. As we have discussed in the previous section, since the theory 830 is unitary, we already know that the anomalous threshold of the triangle diagram does not contribute to (84), therefore also 82 does not contain contributions from this anomalous threshold.

Let us assume that $p_{1}$ is the momentum of the particle corresponding to the initial state, and $p_{2}$ and $p_{3}$ are the momenta of the particles in the final state. For the energy conservation, it will be $p_{1}=p_{2}+p_{3}$. The amplitude of the triangle diagram is

$$
\mathcal{M}\left(p_{h}, \epsilon\right)=-\frac{\lambda^{2}}{2} \int_{\left(\mathcal{C} \times \mathbb{R}^{3}\right)} \frac{i d^{4} k}{(2 \pi)^{4}} \frac{1}{k^{2}-m_{1}^{2}+i \epsilon} \frac{1}{\left(k-p_{1}\right)^{2}-m_{2}^{2}+i \epsilon} \frac{B\left(k, p_{1}, p_{2}, p_{3}\right)}{\left(k-p_{3}\right)^{2}-m_{3}^{2}+i \epsilon},
$$

where $B\left(k, p_{1}, p_{2}, p_{3}\right) \equiv \mathcal{V}\left(p_{1}, k, p-k\right) \mathcal{V}\left(p_{1}-k, p_{2}, p_{3}-k\right) \mathcal{V}\left(p_{3}-k, p_{3}, k\right)$. Since we want this amplitude to be nonzero, we assume $p_{1}^{2}=M^{2}>p_{2}^{2}+p_{3}^{2}$. We also assume that the mass of the internal propagators are different, so that the three propagators can go on shell together. For instance, this happens in the case $m_{1}=m_{2}=m$, and $p_{2}^{2}=p_{3}^{2}=p^{2}>m^{2}+m_{3}^{2}$, when one has an anomalous threshold at 


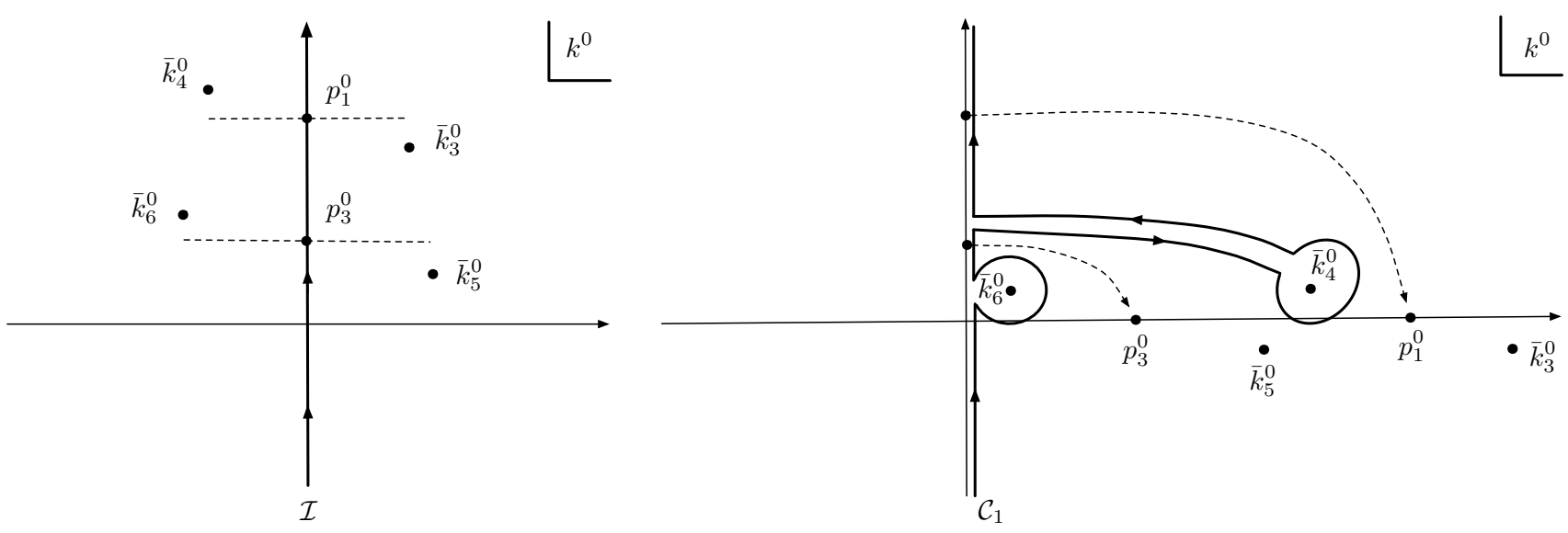

FIG. 9: (Left) We plot the poles $\bar{k}_{3}^{0}, \bar{k}_{4}^{0}, \bar{k}_{5}^{0}, \bar{k}_{6}^{0}$ on the complex $k^{0}$ plane, when the external energies $p_{1}^{0}$ and $p_{3}^{0}$ are purely imaginary. (Right) We plot the same poles when the external energies are moved to their physical real and positive values. The poles $\bar{k}_{4}^{0}$ and $\bar{k}_{6}^{0}$ pass through the imaginary axis for some values of the loop momenta $k$, giving the residues in 90 .

$$
p_{1}^{2}=4 m^{2}-\frac{\left(p^{2}-m^{2}-m_{3}^{2}\right)^{2}}{m_{3}^{2}},
$$

see [10] for details. Anomalous thresholds occur also in other configurations, e.g., in the case $m_{1}=m_{3}=m, m_{2}=2 m$, $p_{2}^{2}=m^{2}, p_{3}^{2}=4 m^{2}$ for $p_{1}^{2}=9 m^{2}$.

Even before starting our calculation, we already know that the anomalous threshold corresponding to the cut diagram with the three internal lines on shell cannot contribute to the imaginary part of $\mathcal{M}$. In fact, we have already shown that $\mathcal{M}-\mathcal{M}^{*}$ contains terms encompassing at most the product of $L+1$ delta functions, namely at most two cut lines in the case of the one-loop triangle diagram under consideration. Therefore, the anomalous threshold corresponding to three cut lines does not contribute to $\mathcal{M}-\mathcal{M}^{*}$. An explicit calculation will show that we have contributions from the same cut diagrams that contribute in the case of the local theory (83), as expected. This is due to the fact that $B\left(k, p_{1}, p_{2}, p_{3}\right)$ has no zeros or poles, thus the local and nonlocal fields (3) and (83) have the same singularity structure.

We know that the integration contour $\left(\mathcal{C} \times \mathbb{R}^{3}\right)$ in $(86)$ is obtained deforming the imaginary axis $\mathcal{I}$ around the poles of the propagators in (86) that pass through it when the energies $p_{1}^{0}, p_{2}^{0}, p_{3}^{0}$ become real. The poles of the first propagator are

$$
\bar{k}_{1,2}^{0}= \pm \sqrt{\vec{k}^{2}+m_{1}^{2}-i \epsilon}
$$

Such poles do not depend on the external energies, and remain always far from the imaginary axis. Indeed, $\bar{k}_{1,2}^{0}$ do not pinch $\mathcal{I}$ and do not contribute to the sum of residues in $(25)$. The poles of the second and third propagators are

$$
\bar{k}_{3,4}^{0}=p_{1}^{0} \pm \sqrt{\left(\vec{k}-\vec{p}_{1}\right)^{2}+m_{2}^{2}-i \epsilon}, \quad \bar{k}_{5,6}^{0}=p_{3}^{0} \pm \sqrt{\left(\vec{k}-\vec{p}_{3}\right)^{2}+m_{3}^{2}-i \epsilon} .
$$

When the external energies are purely imaginary, $\bar{k}_{3}^{0}$ and $\bar{k}_{5}^{0}$ are at the right and $\bar{k}_{4}^{0}$ and $\bar{k}_{6}^{0}$ are at the left of the imaginary axis of the $k^{0}$ plane. When the external energies are moved to their physical real and positive values, such poles move to the right, and it happens that the poles $\bar{k}_{4}^{0}$ and $\bar{k}_{6}^{0}$ pass through the imaginary axis for some values of the loop momenta $k$, see Fig.9. Therefore, the integration contour $\mathcal{C}$ is obtained deforming the imaginary axis $\mathcal{I}$ around the poles $\bar{k}_{4}^{0}$ and $\bar{k}_{6}^{0}$ when such poles pass through $\mathcal{I}$, i.e., when $\Re\left\{\bar{k}_{4}^{0}\right\}>0$ and $\Re\left\{\bar{k}_{6}^{0}\right\}>0$ respectively. 
According to 25, we can express 86 as

$$
\begin{aligned}
\mathcal{M}\left(p_{h}, \epsilon\right)= & -\frac{\lambda^{2}}{2}\left[\int_{\left(\mathcal{I} \times \mathbb{R}^{3}\right)} \frac{i d^{4} k}{(2 \pi)^{4}} \frac{1}{k^{2}-m_{1}^{2}+i \epsilon} \frac{B\left(k, p_{1}, p_{2}, p_{3}\right)}{\left(k-p_{1}\right)^{2}-m_{2}^{2}+i \epsilon} \frac{1}{\left(k-p_{3}\right)^{2}-m_{3}^{2}+i \epsilon}\right. \\
& +\int_{\left(\mathbb{R}^{3}\right)} \frac{i d^{3} k}{(2 \pi)^{4}} 2 \pi i \sigma\left(\Re\left\{\bar{k}_{4}^{0}\right\}\right) \operatorname{Res}\left\{\frac{B\left(k, p_{1}, p_{2}, p_{3}\right)}{k^{2}-m_{1}^{2}+i \epsilon} \frac{1}{\left(k-p_{1}\right)^{2}-m_{2}^{2}+i \epsilon} \frac{1}{\left(k-p_{3}\right)^{2}-m_{3}^{2}+i \epsilon}, \bar{k}_{4}^{0}\right\} \\
& \left.+\int_{\left(\mathbb{R}^{3}\right)} \frac{i d^{3} k}{(2 \pi)^{4}} 2 \pi i \sigma\left(\Re\left\{\bar{k}_{6}^{0}\right\}\right) \operatorname{Res}\left\{\frac{B\left(k, p_{1}, p_{2}, p_{3}\right)}{k^{2}-m_{1}^{2}+i \epsilon} \frac{1}{\left(k-p_{1}\right)^{2}-m_{2}^{2}+i \epsilon} \frac{1}{\left(k-p_{3}\right)^{2}-m_{3}^{2}+i \epsilon}, \bar{k}_{6}^{0}\right\}\right] .
\end{aligned}
$$

The residues in 90 are easily calculated, giving

$$
\begin{aligned}
\operatorname{Res}\{ & \left.\frac{1}{k^{2}-m_{1}^{2}+i \epsilon} \frac{1}{\left(k-p_{1}\right)^{2}-m_{2}^{2}+i \epsilon} \frac{B\left(k, p_{1}, p_{2}, p_{3}\right)}{\left(k-p_{3}\right)^{2}-m_{3}^{2}+i \epsilon}, \bar{k}_{4}^{0}\right\} \\
& =\left.\left(-\frac{B\left(k, p_{1}, p_{2}, p_{3}\right)}{k^{2}-m_{1}^{2}+i \epsilon} \frac{1}{2 \sqrt{\left(\vec{k}-\vec{p}_{1}\right)^{2}+m_{2}^{2}-i \epsilon}} \frac{1}{\left(k-p_{3}\right)^{2}-m_{3}^{2}+i \epsilon}\right)\right|_{k^{0}=\bar{k}_{4}^{0}},
\end{aligned}
$$

and

$$
\begin{aligned}
& \operatorname{Res}\left\{\frac{1}{k^{2}-m^{2}+i \epsilon} \frac{1}{\left(k-p_{1}\right)^{2}-m^{2}+i \epsilon} \frac{B\left(k, p_{1}, p_{2}, p_{3}\right)}{\left(k-p_{3}\right)^{2}-m^{2}+i \epsilon}, \bar{k}_{6}^{0}\right\} \\
& \quad=\left.\left(-\frac{B\left(k, p_{1}, p_{2}, p_{3}\right)}{k^{2}-m^{2}+i \epsilon} \frac{1}{2 \sqrt{\left(\vec{k}-\vec{p}_{3}\right)^{2}+m^{2}-i \epsilon}} \frac{1}{\left(k-p_{1}\right)^{2}-m^{2}+i \epsilon}\right)\right|_{k^{0}=\bar{k}_{6}^{0}},
\end{aligned}
$$

so that the amplitude 90 becomes

$$
\begin{aligned}
& \mathcal{M}\left(p_{h}, \epsilon\right)=-\frac{\lambda^{2}}{2}\left[\int_{\left(\mathcal{I} \times \mathbb{R}^{3}\right)} \frac{i d^{4} k}{(2 \pi)^{4}} \frac{1}{k^{2}-m_{1}^{2}+i \epsilon} \frac{B\left(k, p_{1}, p_{2}, p_{3}\right)}{\left(k-p_{1}\right)^{2}-m_{2}^{2}+i \epsilon} \frac{1}{\left(k-p_{3}\right)^{2}-m_{3}^{2}+i \epsilon}\right. \\
& \left.+\left.\int_{\left(\mathbb{R}^{3}\right)} \frac{i d^{3} k}{(2 \pi)^{4}}(-2 \pi i) \sigma\left(\Re\left\{\bar{k}_{4}^{0}\right\}\right)\left(\frac{B\left(k, p_{1}, p_{2}, p_{3}\right)}{k^{2}-m_{1}^{2}+i \epsilon} \frac{1}{2 \sqrt{\left(\vec{k}-\vec{p}_{1}\right)^{2}+m_{2}^{2}-i \epsilon}} \frac{1}{\left(k-p_{3}\right)^{2}-m_{3}^{2}+i \epsilon}\right)\right|_{k^{0}=\bar{k}_{4}^{0}}\right)\left.\right|_{k^{0}=\bar{k}_{6}^{0}} \\
& +\int_{\left(\mathbb{R}^{3}\right)} \frac{i d^{3} k}{(2 \pi)^{4}}(-2 \pi i) \sigma\left(\Re\left\{\bar{k}_{6}^{0}\right\}\right)\left(-\frac{B\left(k, p_{1}, p_{2}, p_{3}\right)}{k^{2}-m_{1}^{2}+i \epsilon} \frac{1}{\left.2 \sqrt{\left(\vec{k}-\vec{p}_{3}\right)^{2}+m_{3}^{2}-i \epsilon}\right)^{2}-m_{2}^{2}+i \epsilon}\right)
\end{aligned}
$$

Finally, (93) can be recast by means of 46 as

$$
\begin{aligned}
\mathcal{M}\left(p_{h}, \epsilon\right)=-\frac{\lambda^{2}}{2} & {\left[\int_{\left(\mathcal{I} \times \mathbb{R}^{3}\right)} \frac{i d^{4} k}{(2 \pi)^{4}} \frac{1}{k^{2}-m_{1}^{2}+i \epsilon} \frac{B\left(k, p_{1}, p_{2}, p_{3}\right)}{\left(k-p_{1}\right)^{2}-m_{2}^{2}+i \epsilon} \frac{1}{\left(k-p_{3}\right)^{2}-m_{3}^{2}+i \epsilon}\right.} \\
& +\int_{\left(\mathbb{R}^{4}\right)} \frac{i d^{4} k}{(2 \pi)^{4}}(-2 \pi i) \sigma\left(k^{0}\right) \sigma\left(p_{1}^{0}-k^{0}\right) \frac{B\left(k, p_{1}, p_{2}, p_{3}\right)}{k^{2}-m_{1}^{2}+i \epsilon} \frac{\delta\left(\left(k-p_{1}\right)^{2}-m_{2}^{2}\right)}{\left(k-p_{3}\right)^{2}-m_{3}^{2}+i \epsilon} \\
& \left.+\int_{\left(\mathbb{R}^{4}\right)} \frac{i d^{4} k}{(2 \pi)^{4}}(-2 \pi i) \sigma\left(k^{0}\right) \sigma\left(p_{3}^{0}-k^{0}\right) \frac{B\left(k, p_{1}, p_{2}, p_{3}\right)}{k^{2}-m_{1}^{2}+i \epsilon} \frac{\delta\left(\left(k-p_{3}\right)^{2}-m^{2}\right)}{\left(k-p_{1}\right)^{2}-m_{2}^{2}+i \epsilon}\right] .
\end{aligned}
$$

It is easy to see that the first integral in $\sqrt{94}$ is real in the limit $\epsilon \rightarrow 0$, since $k \in \mathcal{I} \times \mathbb{R}^{3}$, indeed the three denominators in the integrand are never zero, and they become real in such a limit. Therefore, such integral does not contribute to the imaginary part of the complex amplitude, and one has

$$
\begin{aligned}
& \mathcal{M}\left(p_{h}, \epsilon\right)-\mathcal{M}\left(p_{h}, \epsilon\right)^{*}= \\
& -\frac{\lambda^{2}}{2} \int_{\left(\mathbb{R}^{4}\right)} \frac{i d^{4} k}{(2 \pi)^{4}}(-2 \pi i)^{2}\left[\sigma\left(k^{0}\right) \sigma\left(p_{1}^{0}-k^{0}\right)\left(\frac{\delta\left(k^{2}-m_{1}^{2}\right)}{\left(k-p_{3}\right)^{2}-m_{3}^{2}+i \epsilon}+\frac{\delta\left(\left(k-p_{3}\right)^{2}-m_{3}^{2}\right)}{k^{2}-m_{1}^{2}+i \epsilon}\right) \delta\left(\left(p_{1}-k\right)^{2}-m_{2}^{2}\right)\right. \\
& \left.+\sigma\left(k^{0}\right) \sigma\left(p_{3}^{0}-k^{0}\right)\left(\frac{\delta\left(k^{2}-m_{1}^{2}\right)}{\left(k-p_{1}\right)^{2}-m_{2}^{2}+i \epsilon}+\frac{\delta\left(\left(k-p_{1}\right)^{2}-m_{2}^{2}\right)}{k^{2}-m_{1}^{2}+i \epsilon}\right) \delta\left(\left(p_{3}-k\right)^{2}-m_{3}^{2}\right)\right] B\left(k, p_{1}, p_{2}, p_{3}\right) .
\end{aligned}
$$




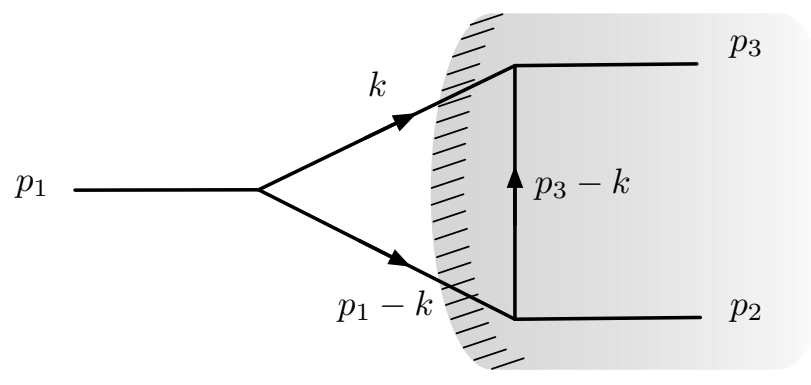

FIG. 10: We here plot the only cut diagram that contributes to $\mathcal{M}-\mathcal{M}^{*}$. In fact, due to the kinematic condition $p_{1}^{2}>p_{2}^{2}+p_{3}^{2}$, the only couple of propagators that can go on shell together is that involving the momenta $k$ and $p_{1}-k$. According to the standard notation, the energy flows from the unshadowed to the shadowed region.

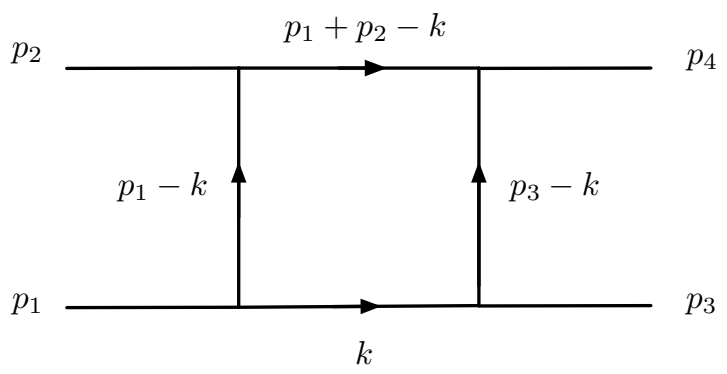

FIG. 11: Box diagram. The momenta $p_{1}$ and $p_{2}$ correspond to the initial state, while $p_{3}$ and $p_{4}$ are the momenta of the final state. Each internal propagator has a different mass, and depending on the choice of such masses, one can have anomalous thresholds with three or four internal momenta on shell.

Finally, we note that the kinematics implies that, since $p_{1}^{2}>p_{2}^{2}+p_{3}^{2}$, the only propagators that can go on shell together are those involving the momenta $k$ and $p_{1}-k$, so that one has

$\mathcal{M}\left(p_{h}, \epsilon\right)-\mathcal{M}\left(p_{h}, \epsilon\right)^{*}=-\frac{\lambda^{2}}{2} \int_{\left(\mathbb{R}^{4}\right)} \frac{i d^{4} k}{(2 \pi)^{4}}(-2 \pi i)^{2} B\left(k, p_{1}, p_{2}, p_{3}\right) \frac{\sigma\left(k^{0}\right) \sigma\left(p_{1}^{0}-k^{0}\right)}{\left(k-p_{3}\right)^{2}-m_{3}^{2}+i \epsilon} \delta\left(k^{2}-m_{1}^{2}\right) \delta\left(\left(p_{1}-k\right)^{2}-m_{2}^{2}\right)$.

From (96) we see that, due to the kinematics, only one of the cut diagrams with two cut lines, more precisely, the one shown in Fig. 10, contributes to $\mathcal{M}-\mathcal{M}^{*}$, while the anomalous threshold of the triangle diagram does not contribute to $\mathcal{M}-\mathcal{M}^{*}$. This is exactly what we were expecting, because, apart from the nonlocal term $B\left(k, p_{1}, p_{2}, p_{3}\right)$, 82 is the same as in local theory. Therefore, it cannot contain other diagrams than those of the the local theory. This example confirms what we stated in the Sec. IV] on the absence of contributions from anomalous thresholds and on the unitarity of the nonlocal theory.

\section{B. Box diagram}

In this section, we study the amplitude of the box diagram in Fig 11 . We assume that $p_{1}$ and $p_{2}$ are the momenta in the initial state, and $p_{3}$ and $p_{4}$ are the momenta in the final state. In a theory with cubic interactions, such momenta corresponds to the momenta of single particles; indeed, they must be on shell. On the contrary, in the case of a quartic interaction, they correspond to the momenta of a couple of particles; indeed, they are not on shell. In both cases, the energy conservation implies $p_{1}+p_{2}=p_{3}+p_{3}$. Here we treat the external momenta as independent variables that do not need to be on shell. The amplitude of the box diagram is

$$
\begin{gathered}
\mathcal{M}\left(p_{h}, \epsilon\right)=-\frac{\lambda^{2}}{2} \int_{\left(\mathcal{C} \times \mathbb{R}^{3}\right)} \frac{i d^{4} k}{(2 \pi)^{4}} \frac{1}{k^{2}-m_{1}^{2}+i \epsilon} \frac{1}{\left(p_{1}-k\right)^{2}-m_{2}^{2}+i \epsilon} \frac{1}{\left(p_{1}+p_{2}-k\right)^{2}-m_{3}^{2}+i \epsilon} \times \\
\frac{1}{\left(p_{3}-k\right)^{2}-m_{4}^{2}+i \epsilon} B\left(k, p_{1}, p_{2}, p_{3}, p_{4}\right),
\end{gathered}
$$


where

$$
B\left(k, p_{1}, p_{2}, p_{3}\right) \equiv \mathcal{V}\left(p_{1}, k, p_{1}-k\right) \mathcal{V}\left(p_{1}-k, p_{2}, p_{1}+p_{2}-k\right) \mathcal{V}\left(p_{1}+p_{2}-k, p_{4}, p_{3}-k\right) \mathcal{V}\left(p_{3}-k, p_{3}, k\right)
$$

We allow the masses of the internal propagators to be different, since we want to consider the leading anomalous threshold in which the four propagators can go on shell at the same time. For instance, if $m_{1}=3 m$ and $m_{2}=$ $m_{3}=m_{4}=m$, the four propagators can go on shell at the same time. Instead, if all the masses are equal, say $m_{1}=m_{2}=m_{3}=m_{4}=m$, only three propagators can go on shell at the same time.

Based on the properties of (82) stated in Sec. IV the imaginary part of the complex amplitude contains contributions coming from cut diagrams with at most $L+1$ cut lines, where $L$ is the number of loops. Therefore, in our one-loop box diagram the contributions to (97) come from diagrams with at most two cut lines, which automatically exclude the anomalous thresholds mentioned above. Since cut diagrams with only one propagator on shell do not give any singularity, because one needs at least two propagators on shell to constrain the integration contour between two poles, we remain with contributions coming only from cut diagrams with two cut lines. Among all the diagrams with two cut lines, the kinematics selects those diagrams allowed by the energy conservation.

Using the usual procedure given by the iterative relations (33) and (34) and the energy conservation $p_{1}+p_{2}=p_{3}+p_{4}$, one has

$$
\begin{gathered}
\mathcal{M}\left(p_{h}, \epsilon\right)-\mathcal{M}\left(p_{h}, \epsilon\right)^{*}=-\frac{\lambda^{2}}{2} \int_{\left(\mathbb{R}^{4}\right)} \sigma\left(k^{0}\right) \frac{i d^{4} k}{(2 \pi)^{4}}(-2 \pi i)^{2} \delta\left(k^{2}-m_{1}^{2}\right) \sigma\left(p_{1}^{0}+p_{2}^{0}-k^{0}\right) \delta\left(\left(p_{1}+p_{2}-k\right)^{2}-m_{3}^{2}\right) \times \\
\frac{1}{\left(p_{1}-k\right)^{2}-m_{2}^{2}+i \epsilon} \frac{1}{\left(p_{3}-k\right)^{2}-m_{4}^{2}+i \epsilon} B\left(k, p_{1}, p_{2}, p_{3}, p_{4}\right),
\end{gathered}
$$

which corresponds to the same cut diagram that contributes in the local case. Therefore, the unitarity of the box diagram is guaranteed.

\section{NONLOCAL QUANTUM GAUGE THEORIES AND GRAVITY}

The Cutkosky rules and the unitarity condition (37) have been derived for a scalar field, but it is quite easy to generalize them to nonlocal gauge theories (NLGT) or nonlocal gravity (NLG). These theories are invariant under gauge or general coordinate transformations that must be treated properly to proof unitarity. In order to prove unitarity, we need to demonstrate the cancellation of the unphysical cuts, which is actually not so different from the local gauge or gravity case. In the scalar field theory, the tensorial structure is trivial, but in NLGT and NLG some of the components of the propagators seem to violate unitarity. However, this is not the case because of the presence of the Faddeev-Popov (FP) ghosts that provide the right cancellations in the amplitude to allow only physical states (physical gauge-invariant polarizations) to go on shell. What we need to use to prove unitarity are the Ward identities that have been derived for a general field theory in Sec. (2.7) of [17] and more recently in [19. Indeed, the proof of the cancellation of unphysical cuts relies only on the gauge or diffeomorphism (or their quantum BRST version) invariance of the action, and it is usually stated at the formal level of the path integral independence of the gauge-fixing term, which, of course, holds here as well.

For NLGT and NLG, we have to sum the contributions coming from gauge bosons and the FP ghosts, but all the amplitudes are analytically continued regardless of the particle species, and the Cutkowsky rules are the same derived for the real scalar field up to an overall tensorial structure.

\section{CONCLUSIONS}

We have proved the Cutkosky rules and the perturbative unitarity for Euclidean nonlocal scalar field and discussed their generalization to gauge and gravitational theories. In short, any scattering amplitude can be computed in Euclidean signature in which all external (and internal) energies are taken purely imaginary. Afterwards, the amplitudes can be analytically continued making the external energies real. The latter operation actually corresponds to integrate along sophisticated paths in the complex plane of the energies circulating in the loops' integrals. It turns out that the analytically continued amplitudes satisfy the Cutkosky rules, and that anomalous thresholds do not contribute to (37), so that the perturbative unitarity of the diagrams is proved. Moreover, we argued that the BRST invariance and the Ward identities imply that all the unphysical cuts present in gauge and gravitational theories cancel with similar contributions coming from the Faddeev-Popov ghosts, and only the physical polarizations can go on shell.

We stress one more time that the fact that the Cutkosky rules are still valid for nonlocal field theories is not surprising. In fact, the Cutkosky result [15] is derived only on the basis of the analysis of the poles of the propagators, 
and the form of the vertices, which can be polynomial or weakly nonlocal, does not play any role in such derivation. In nonlocal theories, the singularities of the amplitudes are still determined by the Landau equations [14], and, therefore, the diagrams in local and nonlocal theories have the same singularities and branch cuts for the same values of the external momenta, which corresponds to the same thresholds. Finally, (37) receives contributions from the same cut diagrams of the local theory (83); indeed, contributions from anomalous thresholds are absent, and the nonlocal theory is unitary.

[1] N. V. Krasnikov, "Nonlocal Gauge Theories," Theor. Math. Phys. 73, 1184 (1987) [Teor. Mat. Fiz. 73, 235 (1987)].

[2] Y. V. Kuz'min, "The Convergent Nonlocal Gravitation (in Russian)," Sov. J. Nucl. Phys. 50, 1011 (1989) [Yad. Fiz. 50, 1630 (1989)].

[3] M. Asorey, J.L. Lope, I.L. Shapiro, Some remarks on high derivative quantum gravity, Int.J.Mod.Phys. A12 (1997) 57115734 .

[4] L. Modesto, "super-renormalizable Quantum Gravity," Phys. Rev. D 86, 044005 (2012) arXiv:1107.2403 [hep-th]].

[5] L. Modesto, "superrenormalizable Multidimensional Quantum Gravity," Astron. Rev. 8, no. 2, 4 (2013) arXiv:1202.3151 [hep-th]].

[6] L. Modesto and L. Rachwal, "Super-renormalizable and finite gravitational theories," Nucl. Phys. B 889, 228 (2014) arXiv:1407.8036 [hep-th]].

[7] L. Modesto and L. Rachwal, "Nonlocal quantum gravity: A review," Int. J. Mod. Phys. D 26, no. 11, 1730020 (2017).

[8] M. Christodoulou, L. Modesto, Reflection positivity in nonlocal gravity, arXiv:1803.08843 [hep-th]. M. Asorey, L. Rachwal, I. L. Shapiro, Unitary Issues in Some Higher Derivative Field Theories, Galaxies 6 (2018) no.1, 23.

[9] M. E. Peskin, D. V. Schroeder, "An Introduction To Quantum Field Theory", Avalon Publishing, 1995.

[10] C. Itzykson, J. B. Zuber, "Quantum Field Theory", Dover publications Inc. 2006, ISBN-10: 0486445682. ISBN-13: 9780486445687.

[11] I. Antoniadis, E. T. Tomboulis, Gauge invariance and unitarity in higher-derivative quantum gravity, Phys. Rev. D 33, 2756 (1986).

[12] L. Modesto and L. Rachwal, "Universally finite gravitational and gauge theories," Nucl. Phys. B 900, 147 (2015) arXiv:1503.00261 [hep-th]].

[13] L. Modesto, M. Piva and L. Rachwal, "Finite quantum gauge theories," Phys. Rev. D 94, no. 2, 025021 (2016) arXiv:1506.06227 [hep-th]].

[14] L. D. Landau, "On analytic properties of vertex parts in quantum field theory", Nuclear Phys. 13, 181 (1959)

[15] R. E. Cutkosky, "Singularities and Discontinuities of Feynman Amplitudes", Journal of Mathematical Physics 1, 429 (1960).

[16] J. C. Taylor, "Analytic Properties of Perturbation Expansions", Phys. Rev. 117, 261 (1960).

[17] I. L. Buchbinder, S. D. Odintsov, I. L. Shapiro, "Effective action in quantum gravity", IOP Publishing Ltd 1992.

[18] M. J. G. Veltman, Unitarity and causality in a renormalizable field theory with unstable particles, Physica 29, 186 (1963); G. t Hooft, M. J. G. Veltman, Diagrammar, NATO Sci. Ser. B 4, 177 (1974).

[19] P. M. Lavrov and I. L. Shapiro, arXiv:1902.04687 [hep-th]. 